\title{
Research into 2D Dynamics and Control of Small Oscillations of a Cross-Beam during Transportation by Two Overhead Cranes
}

\author{
Alexander V. Perig, ${ }^{1}$ Alexander N. Stadnik, ${ }^{2}$ \\ Alexander A. Kostikov, ${ }^{3}$ and Sergey V. Podlesny ${ }^{2}$ \\ ${ }^{1}$ Manufacturing Processes and Automation Engineering Department, Engineering Automation Faculty, \\ Donbass State Engineering Academy, Shkadinova 72, Kramatorsk, Donetsk Region 84313, Ukraine \\ ${ }^{2}$ Department of Technical Mechanics, Engineering Automation Faculty, Donbass State Engineering Academy, \\ Shkadinova 72, Kramatorsk, Donetsk Region 84313, Ukraine \\ ${ }^{3}$ Informatics and Engineering Graphics Department, Engineering Automation Faculty, Donbass State Engineering Academy, \\ Shkadinova 72, Kramatorsk, Donetsk Region 84313, Ukraine \\ Correspondence should be addressed to Alexander V. Perig; olexander.perig@gmail.com
}

Received 13 December 2016; Accepted 5 January 2017; Published 15 February 2017

Academic Editor: Tai Thai

Copyright (C) 2017 Alexander V. Perig et al. This is an open access article distributed under the Creative Commons Attribution License, which permits unrestricted use, distribution, and reproduction in any medium, provided the original work is properly cited.

\begin{abstract}
A new mathematical model of a 3DOF 2D mechanical system "transported cross-beam, two moving bridge cranes" has been proposed. Small system oscillations have been derived through the introduction of Lagrange equations. The numerical estimation of 3DOF system motion has been carried out with equation-based Modelica language. The present article uses the Lagrange method and numerical and optimization methods, realized with JModelica.org and Optimica freeware. The absolute swaying of the crossbeam with respect to the displacement of the two moving bridge cranes was estimated. The phase portraits of the 3DOF system for linear and angular coordinates were presented. An open loop optimal control problem was posed for the motion of the bridge cranes. A "bang-bang" control strategy was implemented for the derivation of an optimal control solution, which enables the travel of two bridge cranes at a prescribed distance for minimum time and minimum swaying of a heavy cross-beam. The derived results of the numerical simulation can be easily practically realized by crane operators with good agreement with simple engineering estimations. The proposed control strategy enables synchronous motion of two bridge cranes with a cross-beam that practically solves the posed problem of unwanted excessive oscillations of a heavy cross-beam during transportation.
\end{abstract}

\section{Background and Introduction}

Current civil engineering technologies often assume a modular concept for the assembly of buildings and other large structures. Individual modules are manufactured at specialized plants and plant departments with further transportation of modular sections to a storage warehouse. Further transportation routes of individual modules lead from the warehouse to the erecting yard at the construction site. Individual modules and modular sections are many times very heavy and bulky objects. Construction plant operation requires simultaneous usage of several cranes, working together at the various stages of transportation of these heavy modules $[1,2]$.
An example of modular unit construction would be the erection of the tower of a wind-powered generator with several modular sections, utilizing two boom cranes working together. This practical case of the two-crane assembly of a wind machine tower requires $3 \mathrm{D}$ turning and tumbling of cylindrical modular sections from a horizontal to a vertical position. In contrast to this example the present article (Figures 1,2, 3, and 6) is focused on the solution of a problem of horizontal transportation of a finished heavy steel beam from a fabrication shop to a storage warehouse.

A shipbuilding example of collaborative working of two or more cranes would be the problem of transportation of a heavy bulk marine engine weighing in the range of 3-3000 tons to the installation site. 


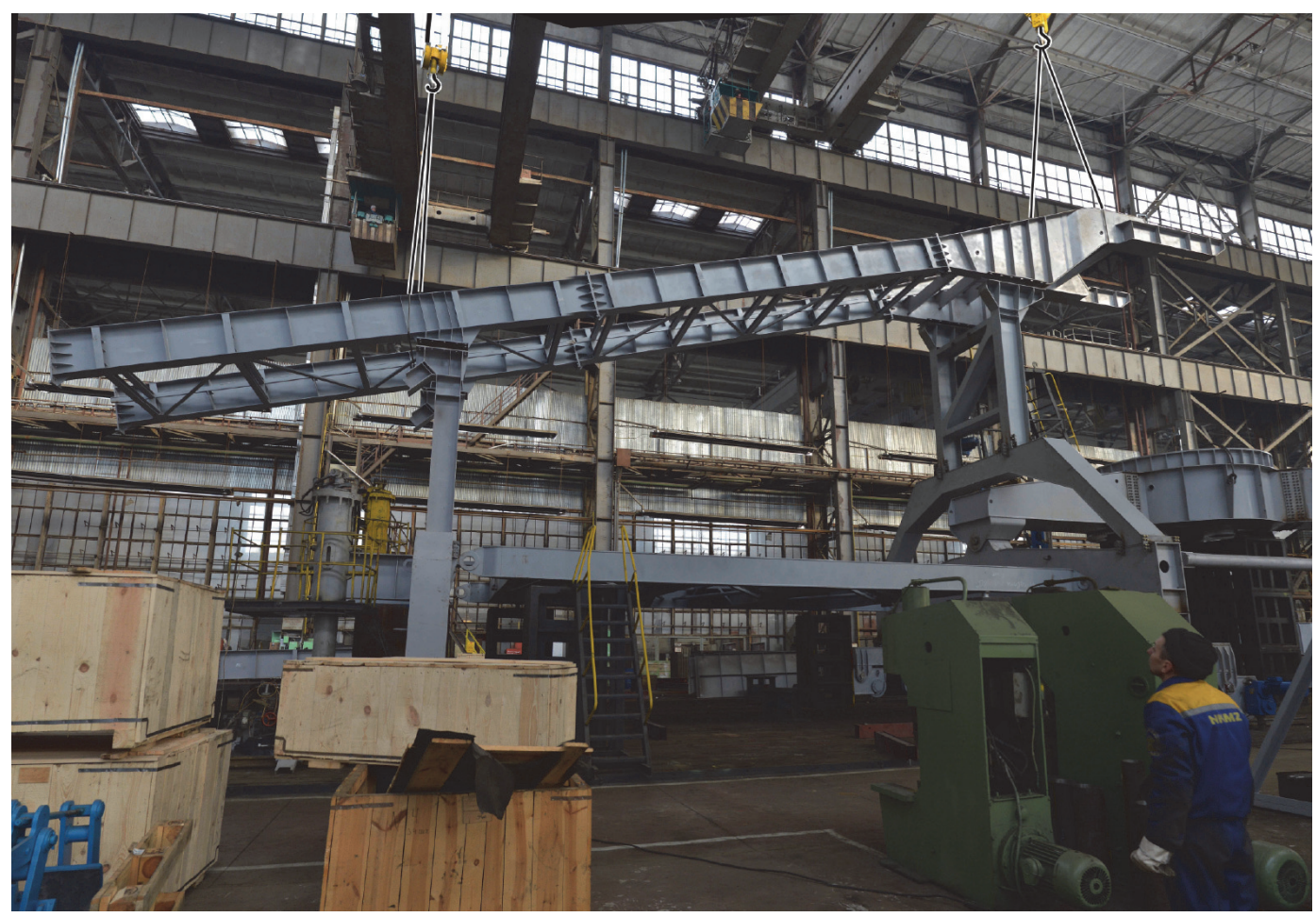

FIGURE 1: Typical plant photos of heavy cross-beam transportation with two overhead cranes.

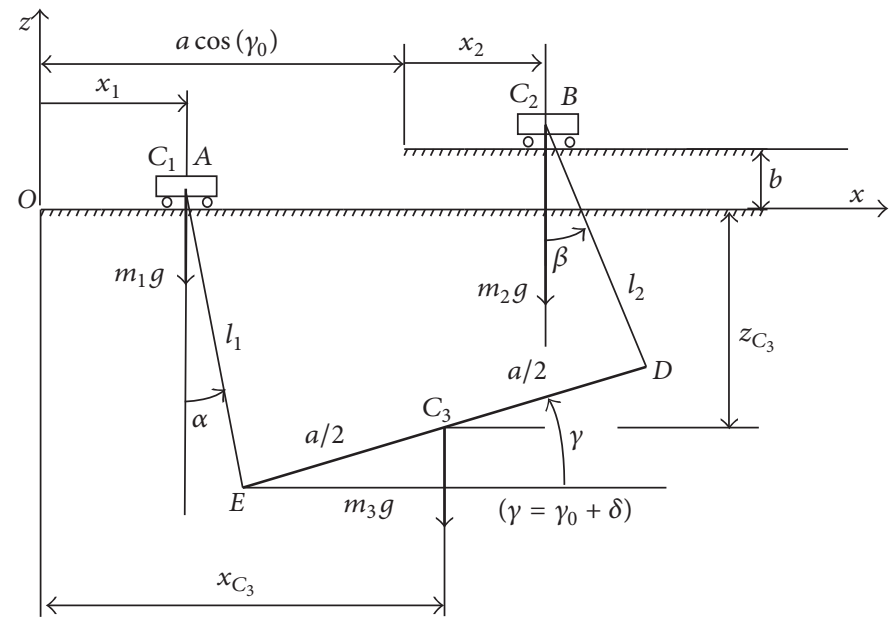

FIgURE 2: The computational scheme for transportation of heavy cross-beam DE by two bridge cranes $A$ and $B$.

Other examples in civil engineering include the liftingand-handling problems of modular building elements of construction, bays of a building, ceiling and floor slab panels, and spandrel walls. Construction problems of roofs, floors, and domes of roofed stadiums and shopping centers require preliminary manufacturing of the heavy bulk trussed frame modules with further transportation to building sites.

It is important to note that attempts to transport heavy bulk stores with a single crane are very undesirable because of the high probability of torsional oscillations of a heavy load. Moreover, usage of two cranes working together for heavy load transportation is a truly natural way for handling displacements in civil and mechanical engineering as nature itself has supplied humans with two hands and two legs for more efficient performance of lifting-and-handling operations.

Overhead crane dynamics has been studied in the research efforts of such scientists as Abdel-Rahman et al. (2003) [1], Deen Ali et al. (2005) [2], Arena et al. (2015) [3], Cartmell et al. (1998) [4], Castelli et al. (2014) [5], Cha et al. (2010) [6], Goodwin (1997) [7], Huang et al. (2015) [8], Lahouar et al. (2009) [9], Pigani and Gallina (2014) [10], 


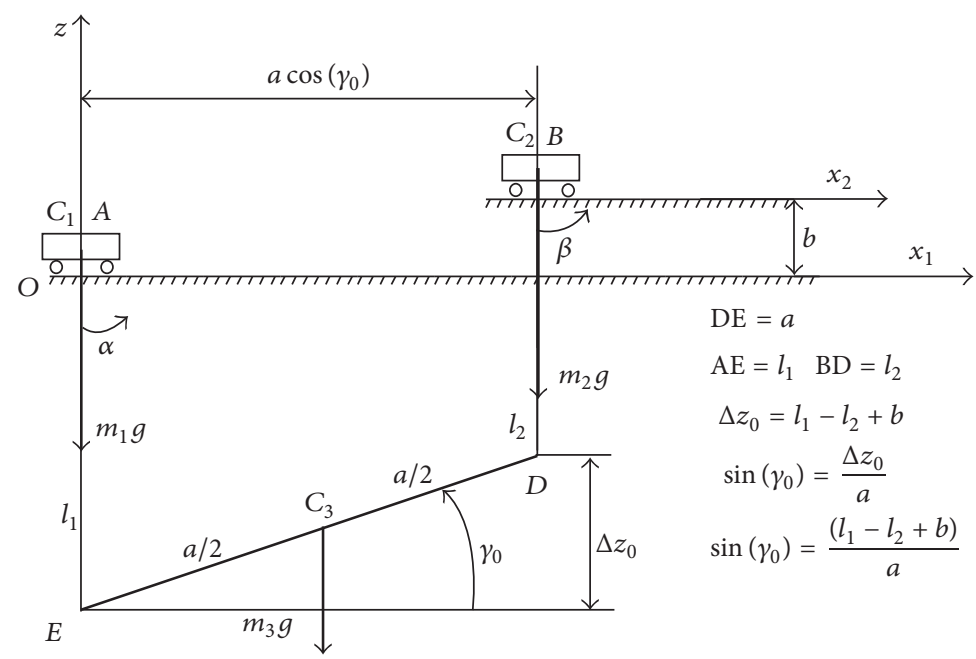

FIgURE 3: The computational scheme of the initial position of mechanical system during transportation of heavy cross-beam DE by two bridge cranes $A$ and $B$.

Sawodny et al. (2002) [11], Smoczek (2014) [12], Zi et al. (2008) [13].

Abdel-Rahman et al. (2003) have discussed mechanical design schemes and mathematical models of gantry cranes and bidirectional gantry cranes with translational displacements of payloads [1]. However payload transportation by two overhead cranes with cross-beam usage is not addressed in [1].

Arena et al. (2015) have proposed several 3D kinematic and dynamic models of container cranes with constant distances between fixed points of supporting rigid and elastic cables [3]. However some disadvantage of Arena's model is associated with a prescribed imposition of geometric constraints on the distances between fixed points for supporting cables. A 2D kinematic model of the present article generalizes Arena's model in the vertical plane [3].

Castelli et al. (2014) have studied a kinetostatic model of a Cartesian cable-suspended robot [5]. A kinematic model of Castelli et al. (2014) is focused on the transportation of a payload using double cables with variable lengths, which provide translational linear motion of the payload without obstacles. There is an analogy between a swaying Cartesian cablesuspended robot (Castelli, 2014) and the swaying cross-beam ED in our problem. However, during transportation the payload may have additional extraneous transverse oscillations. Moreover, successful payload transportation requires a synchronized change of lengths of both supporting cables.

Cha et al. (2010) have studied extra-heavy cargo lifting by two floating cranes with a barge ship connected between the two cranes [6].

Huang et al. (2015) have proposed a new doublependulum mechanical model, describing the transportation of a large payload with distributed mass, which is attached to one trolley by one suspension cable and two rigging cables [8]. Huang et al. (2015) have suppressed payload vibration during transportation by control of trolley acceleration [8]. Mechanical disadvantage of Huang's model is a usage of only one suspension cable. However there are possible strong engineering benefits of using two suspension cables without rigging cables, which is beyond the scope of Huang's research [8].

Kostikov et al. (2016) and Perig et al. (2014) have studied cargo transportation by crane with one slewing pivot point ([14-17]).

Analysis of references [1-18] clearly shows that the dynamics of transportation of the cross-beam by two overhead cranes is not fully addressed in available works [1-18]. This confirms the actuality, relevance, and industrial significance (Figure 1) of the present research.

\section{Research Actuality and Prime Novelty of Research}

The aim of the present research is the dynamic description of the transportation of a heavy cross-beam by two overhead cranes (Figures 1, 2, 3, and 6).

The objective of this research is a $3 \mathrm{DOF} 2 \mathrm{D}$ guided mechanical system "cross-beam $\mathrm{ED}$, cables $\mathrm{AE}$ and $\mathrm{BD}$, moving bridge crane A, moving bridge crane B."

The subject of this research is the study of small oscillations of a heavy cross-beam, induced by the guided motion of two overhead cranes.

The prime novelty of the present research is the introduction of a new mathematical model of a 3DOF 2D mechanical system "cross-beam, two moving bridge cranes" with further dynamic and optimization analysis of small system oscillations and numerical evaluation of guided system motion in the first approach.

The main contribution of the present original research article to the field of engineering transportation is as follows. The 2D problem of horizontal transportation of a heavy steel structure by two bridge cranes, operating at different horizontal levels with different length hoisting cables, has been addressed.

The theoretical novelty of the research is the formulation of differential equations for the motion of a $2 \mathrm{D}$ mechanical 
system "cross-beam, two moving bridge cranes," determination of natural frequencies and periods of oscillations, and the numerical derivation of laws of system motion for coherent initial conditions. An open loop optimal control problem was formulated and solved with JModelica.org and Optimica freeware. The posed optimal control problem numerical solution provides minimization of transportation time for bodies of the mechanical system together with minimal swaying of the heavy bulk load.

The practical sphere of engineering applications of the present transportation problem is in lifting-and-handling machinery and civil engineering.

The plant photo in Figure 1 shows the two bridge cranes with crane operators' cabins, which move along the different horizontal levels. The different lengths of supporting cables $l_{1}$ and $l_{2}$ for holding and carriage of heavy cross-beam by two bridge cranes are clearly observable in Figures 1, 2, 3, and 6.

Figure 1 contains an experimental plant photo of a crossbeam being transported with two independently moving cranes and provides a schematic illustration of the crossbeam inclination angles. The straight inclined line within the cross-beam, which passes through the fixing points of conjunction of the carrying cables' roping to the cross-beam, forms a slope angle $\gamma_{0}$ to the horizon. As a matter of engineering convenience, the present article introduces three interconnected angles $\gamma, \gamma_{0}$, and $\delta$, which uniquely determine the angle of rotation of the cross-beam. The angle $\gamma$ in Figure 2 is the current angle of rotation of a cross-beam ED with respect to the horizontal $x$, where cross-beam ED displacement occurs along the horizontal $x$ direction. Factually the angle $\gamma$ in Figure 2 is a kind of incidence angle, which characterizes the current angle of a cross-beam ED with the direction of the velocity vector of the horizontal displacements of the cranes. It will be shown further that the law of the change of angle $\gamma$ will be a harmonic one with respect to a certain position of static equilibrium of cross-beam ED, which is characterized with angle $\gamma_{0}$ (Figures 1 and 3 ). The new angle $\delta$ in Figure 2 will be introduced as an angle of rotation of a cross-beam ED with respect to the angle $\gamma_{0}$ (Figures 1 and 3) according to the formula $\delta=\gamma-\gamma_{0}$. Therefore the introduction of angle $\delta$ provides a way to select the harmonic component $\delta$ of angle $\gamma$ of cross-beam ED rotation. It is necessary to note that angular velocity of cross-beam ED rotation, which is necessary for calculation of cross-beam kinetic energy, will be determined in the same way using $\delta$ and $\gamma$ angles, as $\omega_{\mathrm{ED}}=$ $d \delta / d t=d \gamma / d t$. It will be shown later that both $\delta$ and $\gamma$ angles are second-order infinitesimal quantities with respect to the small angle $\alpha$ of the deviation of cable 1 from the vertical in Figure 2. Therefore it is possible to consider that the crossbeam ED makes a translational movement with $\omega_{\mathrm{ED}}=$ $d \delta / d t=d \gamma / d t \approx 0$.

\section{Kinematics of the Mechanical System: Geometric Constraints, Coordinates, and Velocities}

The mechanical model of the present problem (Figure 1) is shown in Figures 2, 3, and 6. Dynamic equations for the motion of the mechanical system will be derived using the Lagrange equations $[14,16-18]$ for generalized coordinates $x_{1}$, $x_{2}$, and $\alpha$. So it is necessary to determine kinetic and potential energies of the mechanical system as functions of our chosen generalized coordinates. For this purpose we have to calculate the coordinates of bodies $A, B$, and material point $C_{3}$ of the cross-beam body DE.

The Cartesian coordinates of point $B$, which coincides with the point $C_{2}$, are as follows:

$$
\begin{aligned}
& x_{B}=a \cos \left(\gamma_{0}\right)+x_{2} ; \\
& z_{B}=b \\
& x_{B}=x_{1}+l_{1} \cdot \sin (\alpha)+a \cdot \cos (\gamma)-l_{2} \cdot \sin (\beta) ; \\
& z_{B}=-l_{1} \cdot \cos (\alpha)+a \cdot \sin (\gamma)+l_{2} \cdot \cos (\beta) .
\end{aligned}
$$

System (1) of transcendental equations, which determine the Cartesian coordinates of point $B$, contains variables $x_{1}$; $x_{2}$; and $\alpha$. It is necessary to solve system (1) with respect to the unknown angles $\beta$ and $\gamma$, that is, to derive $\beta=f_{\beta}\left(x_{1}, x_{2}, \alpha\right)$ and $\gamma=f_{\gamma}\left(x_{1}, x_{2}, \alpha\right)$ for $\gamma=\gamma_{0}+\delta$, where the current angle $\delta$ is counted off from the initial inclination angle $\gamma_{0}$ in the direction of cross-beam ED motion in Figures 2 and 3. The simplest way to solve system (1) with respect to the $\beta, \gamma$, and $\delta$ is usage of a small angle assumption (Appendix A-D).

In order to calculate the initial inclination angle $\gamma_{0}$ (Figures 1 and 3) of cross-beam ED with a horizon $x$ it is possible to introduce an additional geometric parameter $\Delta z_{0}=a \cdot \sin \left(\gamma_{0}\right)$ in Figure 3 by substitution of the initial time moment $t_{0}=0(\mathrm{~s})$ into the second lower equations of system (1), which yields the following expression $z_{B}=b=(-1) \cdot l_{1}$. $1+a \cdot \sin \left(\gamma_{0}\right)+l_{2} \cdot 1$, and $\Delta z_{0}=a \cdot \sin \left(\gamma_{0}\right)=b+l_{1}-l_{2}$ or the sinus of $\gamma_{0}$ angle is $\sin \left(\gamma_{0}\right)=\left(b+l_{1}-l_{2}\right) / a=\Delta z_{0} / a$ (Figure 3$)$. These reasons confirm alternative results in formulae (A.2)-(A.4).

Equation (A.12) establishes a simplified linear dependence between swing angles $\alpha$ and $\beta$. So one gets from (A.12) that the small angle $\beta$ in Figure 2 can be estimated on the basis of (A.12) in the following way:

$$
\beta \approx\left(\frac{1}{l_{2}}\right) \cdot\left(x_{1}-x_{2}+l_{1} \alpha\right) .
$$

Equation (2) determines the swing angle $\beta$ as the simplified linear function of the variables $x_{1}, x_{2}, \alpha$; that is, $\beta=$ $f_{\beta}\left(x_{1}, x_{2}, \alpha\right)$.

The rate of change of the swing angle $\beta$, that is, the small angular velocity $\omega_{\beta}=(d \beta / d t)$, can be estimated on the basis of (2) in the following way:

$$
\left(\frac{d \beta}{d t}\right) \approx\left(\frac{1}{l_{2}}\right) \cdot\left(\left(\frac{d x_{1}}{d t}\right)-\left(\frac{d x_{2}}{d t}\right)+l_{1}\left(\frac{d \alpha}{d t}\right)\right) .
$$

Equation (B.9) determines the small inclination angle $\delta$ to be a simplified linear function of the variables $x_{1}, x_{2}, \alpha$; that is, $\delta=f_{\delta}\left(x_{1}, x_{2}, \alpha\right)$. Algebraic expression (B.9) accurately proves the correctness of statement (A.9) concerning the second order of infinitely small $\delta$ (infinitesimality of $\delta=O\left(\alpha^{2}\right)$ ) for a small inclination angle $\delta$, which determines the current 
inclination of the cross-beam ED in Figure 2. Equation (B.9) allows the development of an alternative approximate expression for the small angle $\gamma$ for cross-beam ED inclination in Figure 2:

$$
\begin{aligned}
\gamma= & \gamma_{0}+\delta \approx \gamma_{0} \\
& +\left(\frac { 1 } { ( 2 \cdot a \cdot l _ { 2 } \cdot \operatorname { c o s } ( \gamma _ { 0 } ) ) } \left(\left(x_{1}-x_{2}+l_{1} \alpha\right)^{2}-l_{1} \cdot l_{2}\right.\right. \\
& \left.\left.\cdot \alpha^{2}\right)\right) .
\end{aligned}
$$

The derived alternative equations (B.9) and (4) provide a way for the estimation of the value of the angular velocity $\omega_{\delta}=(d \delta / d t)=\omega_{\gamma}=(d \gamma / d t)$ by the following expression:

$$
\begin{aligned}
& \left(\frac{d \gamma}{d t}\right)=\left(\frac{d \delta}{d t}\right) \approx \frac{1}{\left(a \cdot l_{2} \cdot \cos \left(\gamma_{0}\right)\right)}\left(\left(x_{1}-x_{2}+l_{1} \alpha\right)\right. \\
& \cdot\left(\left(\frac{d x_{1}}{d t}\right)-\left(\frac{d x_{2}}{d t}\right)+l_{1}\left(\frac{d \alpha}{d t}\right)\right)-l_{1} \cdot l_{2} \cdot \alpha \\
& \left.\cdot\left(\frac{d \alpha}{d t}\right)\right) .
\end{aligned}
$$

Algebraic expression (5) also proves the correctness of statement (A.9) concerning the second order of infinitely small $\omega_{\delta}=\omega_{\gamma}$ (infinitesimality of $\omega_{\delta}=(d \delta / d t)=\omega_{\gamma}=$ $\left.(d \gamma / d t)=O\left(\alpha^{2}\right)\right)$ for the angular velocity of the cross-beam ED in Figure 2.

After simplifications (C.4) yields that

$$
\begin{aligned}
z_{C_{3}} \approx & \left(\left(\frac{1}{2}\right) \cdot l_{1}\right) \cdot \alpha^{2} \\
& +\frac{1}{\left(4 \cdot l_{2}\right)}\left(\left(x_{1}-x_{2}+l_{1} \alpha\right)^{2}-l_{1} \cdot l_{2} \cdot \alpha^{2}\right) \\
& +\left(\left(\frac{1}{2}\right) \cdot\left(b-l_{1}-l_{2}\right)\right) .
\end{aligned}
$$

\section{Dynamics of the Mechanical System: Potential and Kinetic Energies, Generalized Forces, and Lagrange Equations}

The potential energy for the mechanical system in Figure 2 can be calculated as

$$
\Pi=\left(m_{3} g\right) \cdot z_{C_{3}} .
$$

Substitution of approximated expression (6) into (7) yields

$$
\begin{aligned}
\Pi & \approx\left(m_{3} g\right) \cdot\left(\left(\left(\frac{1}{2}\right) \cdot l_{1}\right) \cdot \alpha^{2}\right. \\
& +\frac{1}{\left(4 \cdot l_{2}\right)}\left(\left(x_{1}-x_{2}+l_{1} \alpha\right)^{2}-l_{1} \cdot l_{2} \cdot \alpha^{2}\right) \\
& \left.+\left(\left(\frac{1}{2}\right) \cdot\left(b-l_{1}-l_{2}\right)\right)\right) .
\end{aligned}
$$

Estimation of potential energy enables calculation of the generalized forces $Q_{x_{1}}, Q_{x_{2}}$, and $Q_{\alpha}$, which can be calculated as partial derivatives of potential energy $\Pi$ with respect to the general coordinates $x_{1}, x_{2}$, and $\alpha$ (Appendix E). Substitution of (E.2), (E.4), and (E.9) into a one-column matrix yields the following vector of generalized forces:

$$
\begin{aligned}
\{\mathbf{Q}\} \approx & \left(m_{3} g\right) \cdot \frac{1}{\left(2 \cdot l_{2}\right)} \\
& \cdot\left(\begin{array}{c}
-x_{1}+x_{2}-l_{1} \alpha \\
x_{1}-x_{2}+l_{1} \alpha \\
-\left(l_{1} \cdot l_{2}+l_{1}^{2}\right) \cdot \alpha-x_{1} \cdot l_{1}+x_{2} \cdot l_{1}
\end{array}\right) .
\end{aligned}
$$

Simplification of (F.7) results in the following approximated expression:

$$
\begin{aligned}
T \approx & \left(\frac{1}{2}\right) \cdot\left(m_{1}+m_{3}\right) \cdot\left(\frac{d x_{1}}{d t}\right)^{2}+\left(\frac{1}{2}\right) \cdot m_{2} \\
& \cdot\left(\frac{d x_{2}}{d t}\right)^{2}+\left(\frac{1}{2}\right) \cdot m_{3} \cdot l_{1}^{2} \cdot\left(\frac{d \alpha}{d t}\right)^{2}+m_{3} \cdot l_{1} \\
& \cdot\left(\frac{d x_{1}}{d t}\right) \cdot\left(\frac{d \alpha}{d t}\right)
\end{aligned}
$$

The matrix form of the left-hand sides of Lagrange equations (G.1), (G.7), and (G.13) or (G.5), (G.11), and (G.17) yields the following matrix expression:

$$
\begin{aligned}
\frac{d}{d t}\left(\frac{\partial T}{\partial \dot{\mathbf{q}}}\right)-\frac{\partial T}{\partial \mathbf{q}} \approx & \left(\begin{array}{ccc}
\left(m_{1}+m_{3}\right) & 0 & \left(m_{3} \cdot l_{1}\right) \\
0 & m_{2} & 0 \\
\left(m_{3} \cdot l_{1}\right) & 0 & \left(m_{3} \cdot l_{1}^{2}\right)
\end{array}\right) \\
& \left(\begin{array}{c}
\left(\frac{d^{2} x_{1}}{d t^{2}}\right) \\
\left(\frac{d^{2} x_{2}}{d t^{2}}\right) \\
\left(\frac{d^{2} \alpha}{d t^{2}}\right)
\end{array}\right)-0 .
\end{aligned}
$$

The vector form of Lagrange equations is as follows:

$$
\frac{d}{d t}\left(\frac{\partial T}{\partial \dot{\mathbf{q}}}\right)-\frac{\partial T}{\partial \mathbf{q}}=\{\mathbf{Q}\}
$$

where the left-hand side of (12) is determined by matrix product (11) and the right-hand side of (12) is determined by the vector-column (9). 


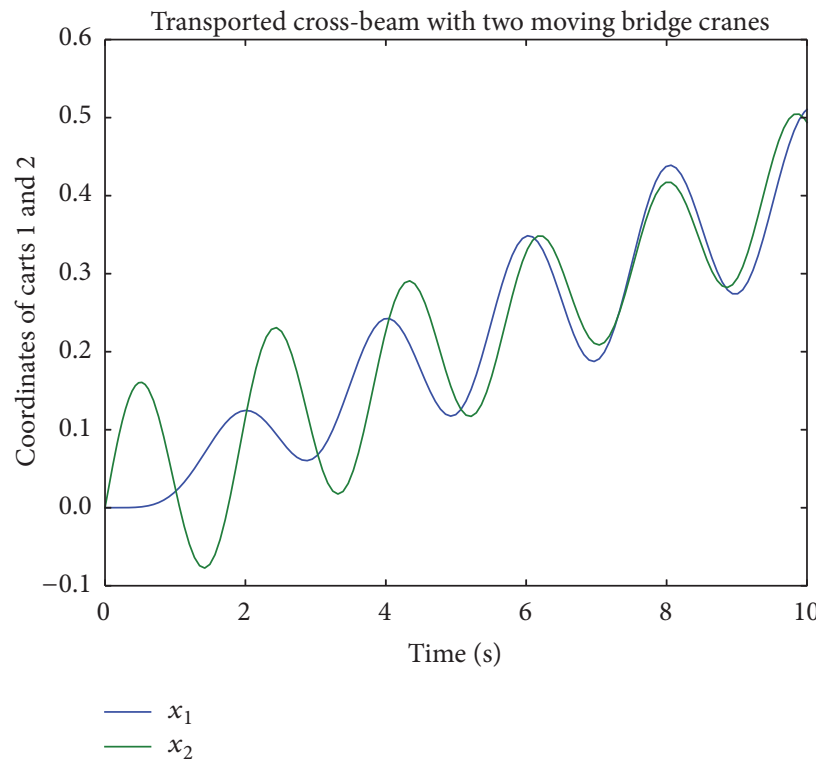

(a)

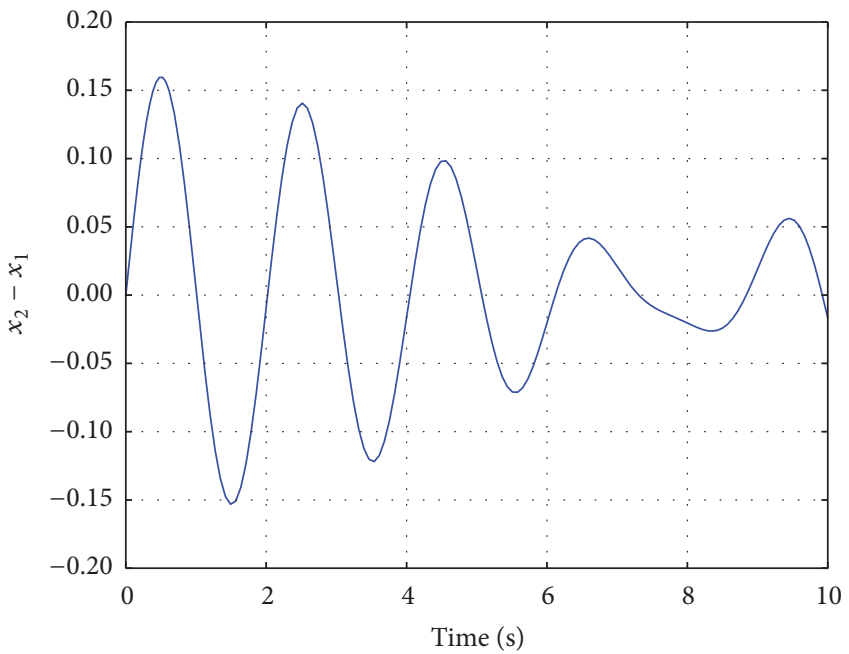

(b)

FIGURE 4: JModelica.org-derived numerical plots for linear coordinates $x_{1}=x_{1}(t) ; x_{2}=x_{2}(t)(\mathrm{a})$; and $x_{2}-x_{1}=x_{2}(t)-x_{1}(t)(\mathrm{b})$, computed for system (13) with nonzero initial velocities $V_{B}(0)=0.5(\mathrm{~m} / \mathrm{s})$ and $\omega_{\beta}(0) \approx-0.083(\mathrm{rad} / \mathrm{s})$.

Substitution of (11) and (9) into (12) results in the following matrix form of Lagrange equations for the motion of the system in Figures 2 and 3:

$$
\left(\begin{array}{ccc}
\left(m_{1}+m_{3}\right) & 0 & \left(m_{3} \cdot l_{1}\right) \\
0 & m_{2} & 0 \\
\left(m_{3} \cdot l_{1}\right) & 0 & \left(m_{3} \cdot l_{1}^{2}\right)
\end{array}\right) \cdot\left(\begin{array}{c}
\left(\frac{d^{2} x_{1}}{d t^{2}}\right) \\
\left(\frac{d^{2} x_{2}}{d t^{2}}\right) \\
\left(\frac{d^{2} \alpha}{d t^{2}}\right)
\end{array}\right)
$$

Derived equation (G.6), (G.12), (G.18), or (13) allows the determination of natural periods of system oscillations, natural frequencies of mechanical system, and deriving motion equations for each of the three bodies of studied mechanical system for different initial conditions in Figures 1-3. The numerical solution of Lagrange equation (G.6), (G.12), (G.18), or (13) enables the determination of motion patterns and formulating applied engineering recommendations concerning motion of the system.

\section{First Computational Example of Numerical Integration of Derived Lagrange Equations for the Studied Mechanical System with JModelica.org Freeware}

Both Figure 3 and formulae (2)-(3) yield that at the initial time moment $t_{0}=0(\mathrm{~s}),(2)$ results in $\beta_{0} \approx\left(\left(x_{1}\right)_{0}-\left(x_{2}\right)_{0}+l_{1}\right.$. $\left.\left(\alpha_{0}\right)\right) / l_{2}=0$. The time derivative of this expression provides an analogical algebraic equation for initial velocities $\omega_{\beta}(0)=$ $d\left(\beta_{0}\right) / d t \approx\left(\left(V_{1}\right)_{0}-\left(V_{2}\right)_{0}+l_{1} \cdot\left(\left(d\left(\alpha_{0}\right)\right) / d t\right)\right) / l_{2}$ of system in Figures 1-3, which results in the following equations: $l_{2} \cdot \omega_{\beta}(0)=$ $\left(V_{1}\right)_{0}-\left(V_{2}\right)_{0}+l_{1} \cdot \omega_{\alpha}(0)$ and $\omega_{\alpha}(0)=d\left(\alpha_{0}\right) / d t=\left(\left(V_{2}\right)_{0}-\left(V_{1}\right)_{0}+\right.$ $\left.l_{2} \cdot \omega_{\beta}(0)\right) / l_{1}=\left(\left(V_{B}\right)_{0}-\left(V_{A}\right)_{0}+l_{2} \cdot \omega_{\beta}(0)\right) / l_{1}$. The numerical JModelica.org-enhanced solution of the posed problem in Figures 4 and 5 of the present article was derived in the typical engineering case $\left(V_{2}\right)_{0}=\left(V_{B}\right)_{0} \neq 0$ of right-hand side motion of the crane $B$ along $x$ direction, when the system motion started from the system equilibrium position with zeroinitial-values of three generalized initial coordinates $\left(x_{1}\right)_{0}=$ $\left(x_{2}\right)_{0}=\left(\alpha_{0}\right)=0$. Assumption of $\left(V_{1}\right)_{0}=\left(V_{A}\right)_{0}=0(\mathrm{~m} / \mathrm{s})$ and $\left(V_{2}\right)_{0}=\left(V_{B}\right)_{0} \neq 0$ yields $\omega_{\beta}(0)=d\left(\beta_{0}\right) / d t \approx\left(l_{1} \cdot \omega_{\alpha}(0)-\right.$ $\left.\left(V_{2}\right)_{0}\right) / l_{2}$. Additional assumption $\omega_{\alpha}(0)=0$ results in $\omega_{\beta}(0)=$ $d\left(\beta_{0}\right) / d t \approx\left((-1) \cdot\left(V_{2}\right)_{0}\right) / l_{2}=\left((-1) \cdot\left(V_{B}\right)_{0}\right) / l_{2}$. The derivation of numerical solutions of inverse dynamic problems for mechanical system in Figures 1-3 requires the assignment of different initial values of system coordinates and velocities, which satisfy the abovementioned constraint equations for initial coordinates $\beta_{0} \approx\left(\left(x_{1}\right)_{0}-\left(x_{2}\right)_{0}+l_{1} \cdot\left(\alpha_{0}\right)\right) / l_{2}=0$ and initial velocities $\omega_{\alpha}(0)=d\left(\alpha_{0}\right) / d t=\left(\left(V_{2}\right)_{0}-\left(V_{1}\right)_{0}+l_{2} \cdot \omega_{\beta}(0)\right) / l_{1}=$ $\left(\left(V_{B}\right)_{0}-\left(V_{A}\right)_{0}+l_{2} \cdot \omega_{\beta}(0)\right) / l_{1}$. These questions concerning the influence of initial conditions were properly addressed by the 


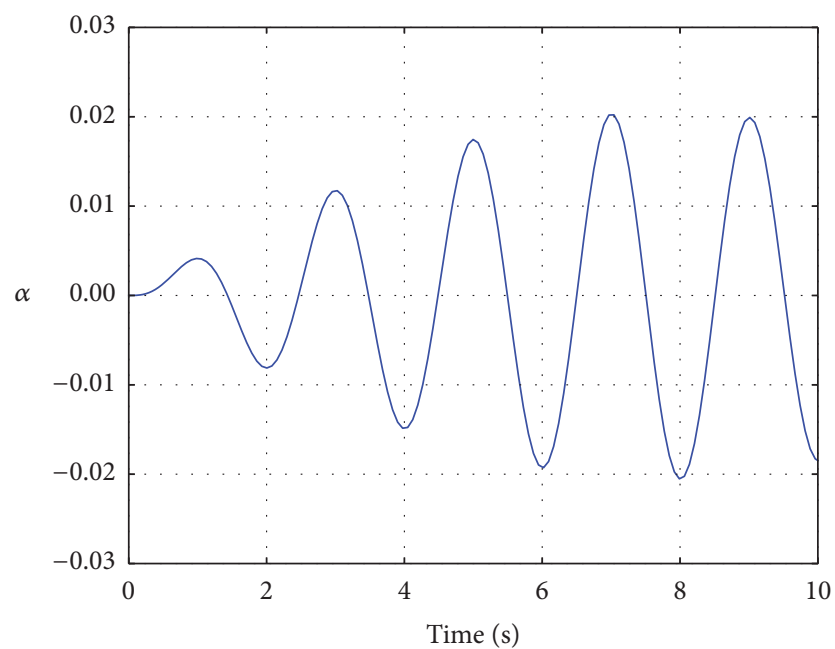

(a)

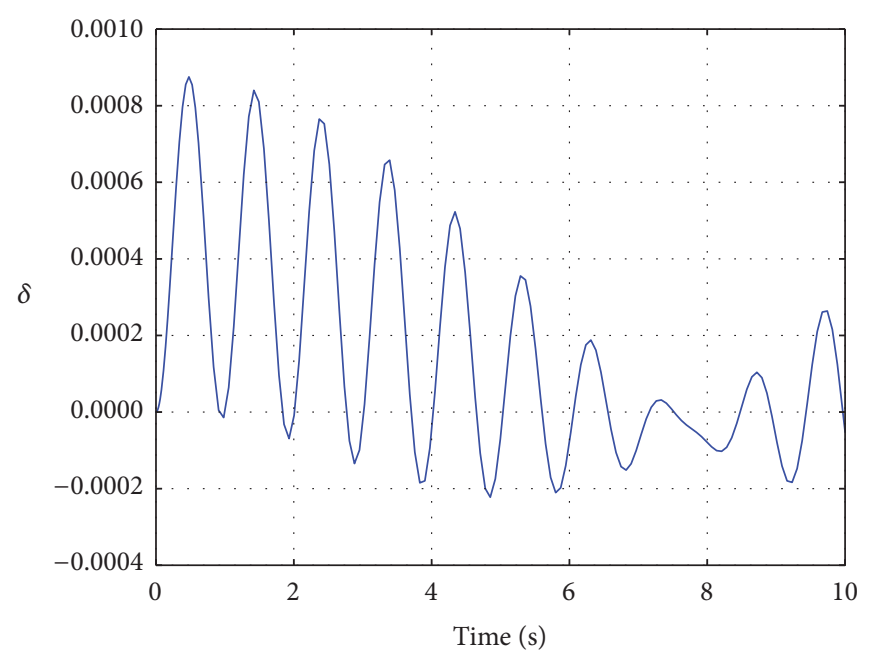

(c)

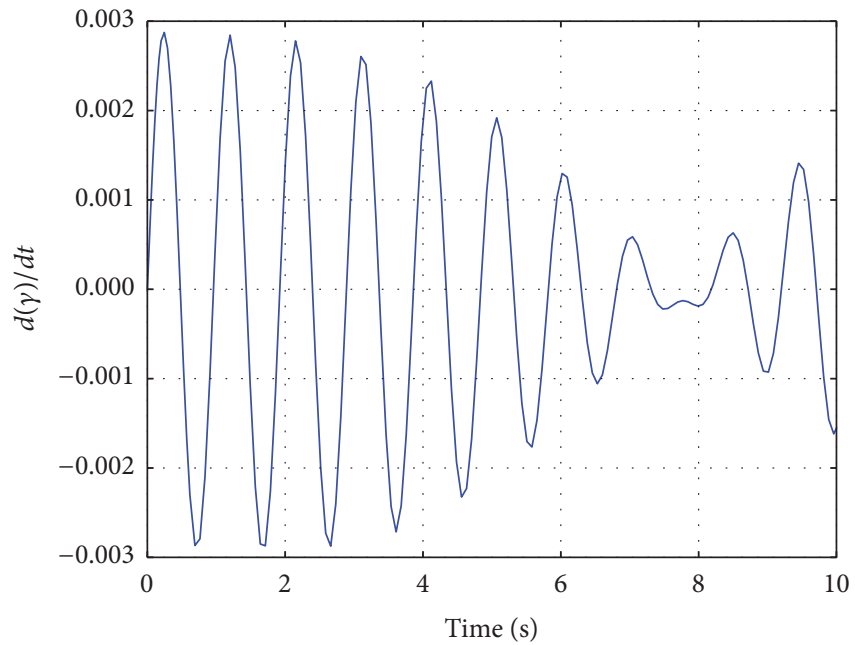

(e)

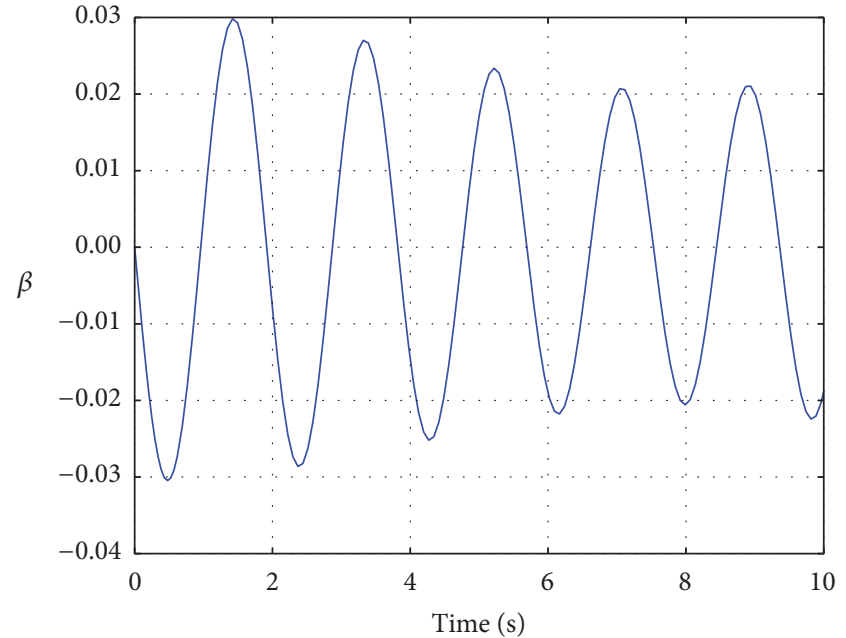

(b)

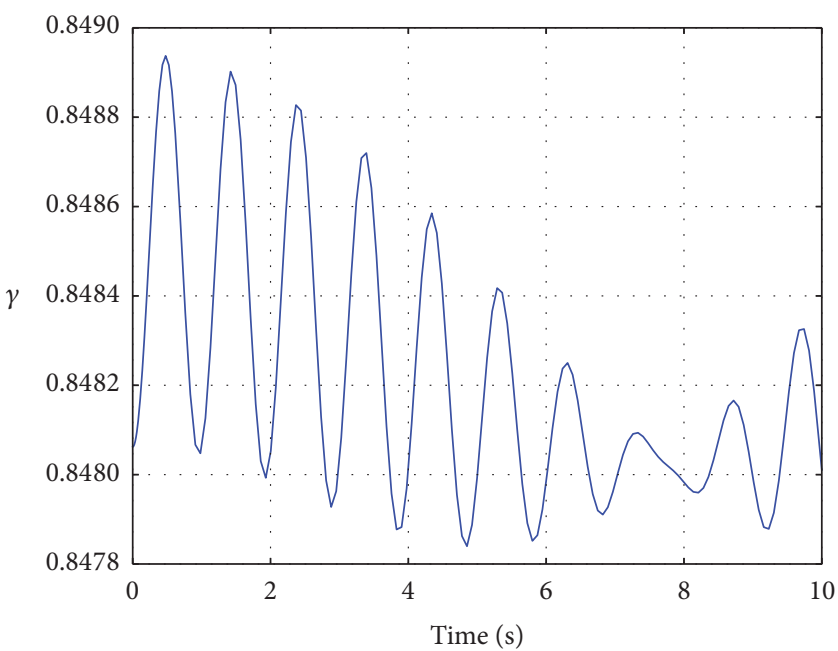

(d)

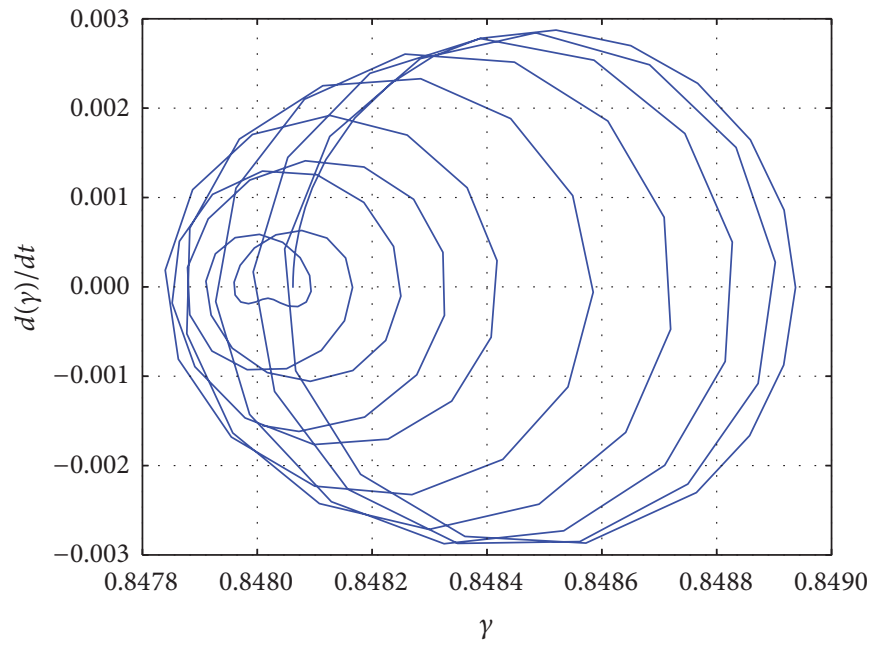

(f)

FIGURE 5: JModelica.org-derived numerical plots for angular coordinates $\alpha=\alpha(t)$ (a); $\beta=\beta(t)(\mathrm{b}) ; \delta=\delta(t)$ (c); $\gamma=\gamma(t)$ (d); $\omega_{\gamma}=\omega_{\gamma}(t)=$ $(d(\gamma(t)) / d t)(\mathrm{e})$; and $\omega_{\gamma}=\omega_{\gamma}(\gamma)$; that is, $(d(\gamma(t)) / d t)=f(\gamma)(\mathrm{f})$, computed for system (13) with nonzero initial velocities $V_{B}(0)=0.5(\mathrm{~m} / \mathrm{s})$ and $\omega_{\beta}(0) \approx-0.083(\mathrm{rad} / \mathrm{s})$. 


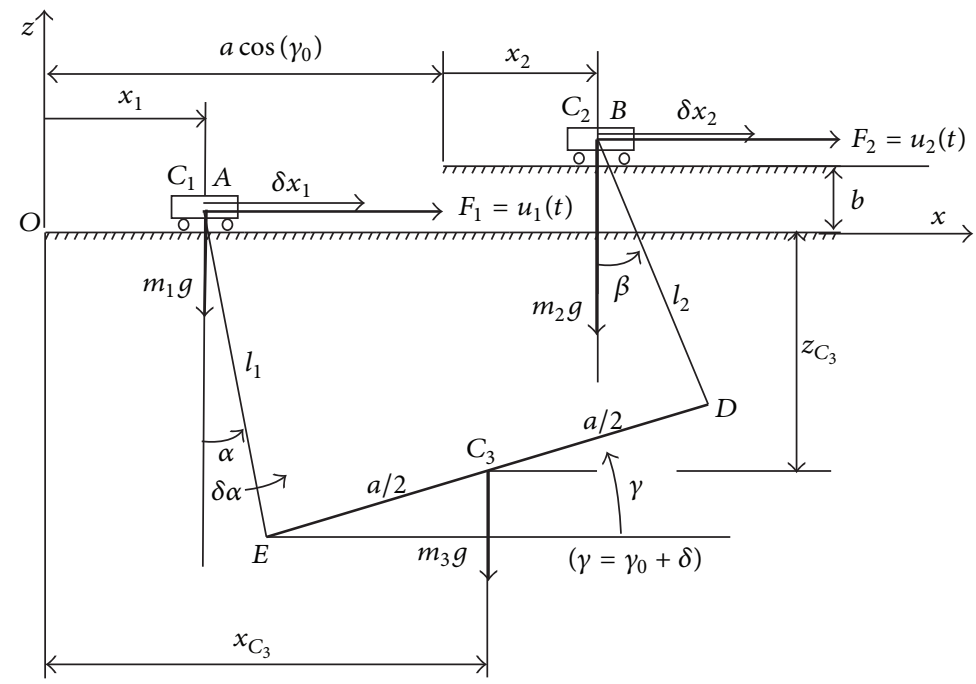

FIGURE 6: The computational scheme for transportation of heavy cross-beam DE by two guided bridge cranes $A$ and $B$ with applied control forces to carts $A$ and $B$ with forces' magnitudes $F_{1}=u_{1}(t)$ and $F_{2}=u_{2}(t)$.

authors in the present research and one of the typical cases is shown in numerical plots in Figures 4 and 5.

It is useful to get the numerical solution of system (13) of Lagrange equations (G.6), (G.12), and (G.18) with freeware code JModelica.org $[14,15,19]$ for the following numerical values of parameters of the mechanical system in Figure 2: gravity acceleration is $g=9.81\left(\mathrm{~m} / \mathrm{s}^{2}\right)$; masses of movable carts $A$ and $B$ are $m_{1}=m_{2}=1000(\mathrm{~kg})$; mass of the cross-beam ED is $m_{3}=10000(\mathrm{~kg})$; length of the left cable AE is $l_{1}=6(\mathrm{~m})$; length of the right cable BD is $l_{2}=5(\mathrm{~m})$; vertical distance between the carts is $b=2(\mathrm{~m})$; length of the cross-beam ED is $a=4(\mathrm{~m})$.

The initial conditions for system (13) of Lagrange equations (G.6), (G.12), and (G.18) are as follows: it is possible to assume that at the initial time moment $t=0$ (s) the right upper cart $B$ in Figure 2 moves rectilinearly to the right; that is, initial coordinate of the left cart $A$ is $x_{1}(0)=0(\mathrm{~m})$; initial velocity of the left cart $A$ is $V_{A}(0)=\left(d\left(x_{1}(0)\right) / d t\right)=0(\mathrm{~m} / \mathrm{s})$; initial coordinate of the right cart $B$ is $x_{2}(0)=0(\mathrm{~m})$; initial velocity of the right cart $B$, which moves to the right, is $\left(V_{2}\right)_{0}=V_{B}(0)=\left(d\left(x_{2}(0)\right) / d t\right)=0.5(\mathrm{~m} / \mathrm{s}) \neq 0$; initial swing angle of the left rope AE is $\alpha(0)=0(\mathrm{rad})$; initial angular velocity of the left cable $\mathrm{AE}$ is $\omega_{\alpha}(0)=(d(\alpha(0)) / d t)=$ $0(\mathrm{rad} / \mathrm{s})$. The abovementioned constraint equations yield that $\omega_{\beta}(0)=d\left(\beta_{0}\right) / d t \approx\left((-1) \cdot\left(V_{2}\right)_{0}\right) / l_{2}=\left((-1) \cdot\left(V_{B}\right)_{0}\right) / l_{2}$ $=(-1) \cdot 0.5(\mathrm{~m} / \mathrm{s}) / 6(\mathrm{~m}) \approx-0.083(\mathrm{rad} / \mathrm{s})$. The corresponding computational results are shown in Figures 4 and 5.

Computational Figures 4 and 5 show the increase of carts' coordinates with respect to motion time $t$. The computational curves in Figures 4 and 5 also show the additional harmonic oscillations. Computational dependencies of the angular coordinates in Figure 5 are harmonic curves. The computational amplitudes in Figure 5 confirm the correctness of small angle assumption in the present article.

Computational Figure 5(f) shows an unstable focus at the phase plane $(\gamma(t), d(\gamma(t)) / d t)$ that means a certain increase in amplitude during cross-beam oscillations. However it is possible to neglect some increase of $\gamma$ amplitude by taking into account the fact that both $\gamma(t)$ and $(d(\gamma(t)) / d t)$ are secondorder infinitesimal quantities. So from an engineering standpoint some small increase in $\gamma(t)$ during a cross-beam transportation is negotiable and not essential. Moreover Figure 5(f) shows the necessity of application of additional damping devices for suppression of a $\gamma(t)$ oscillations.

\section{Dynamics of the Guided Mechanical System}

The formulation of the optimal control problem for guided motion of bridge cranes $A$ and $B$ requires the application of two independent external control forces to moving carts $A$ and $B$ with magnitudes $F_{1}=u_{1}(t)[\mathrm{N}]$ and $F_{2}=u_{2}(t)[\mathrm{N}]$ of variable forces in Figure 6.

Substitution of (I.9), (I.14), and (I.19) into the one-column matrix yields the following vector of generalized forces for the guided mechanical system:

$$
\begin{aligned}
\left\{\mathbf{Q}_{\delta}\right\} \approx & \left(m_{3} g\right) \cdot \frac{1}{\left(2 \cdot l_{2}\right)} \\
& \cdot\left(\begin{array}{c}
-x_{1}+x_{2}-l_{1} \alpha \\
x_{1}-x_{2}+l_{1} \alpha \\
-\left(l_{1} \cdot l_{2}+l_{1}^{2}\right) \cdot \alpha-x_{1} \cdot l_{1}+x_{2} \cdot l_{1}
\end{array}\right) \\
& +\left(\begin{array}{c}
F_{1} \\
F_{2} \\
0
\end{array}\right) .
\end{aligned}
$$

Substitution of (11) and (14) into (12) results in the following matrix form of the Lagrange equations for the motion of the guided mechanical system in Figure 6: 


$$
\begin{aligned}
& \left(\begin{array}{ccc}
\left(m_{1}+m_{3}\right) & 0 & \left(m_{3} \cdot l_{1}\right) \\
0 & m_{2} & 0 \\
\left(m_{3} \cdot l_{1}\right) & 0 & \left(m_{3} \cdot l_{1}^{2}\right)
\end{array}\right) \cdot\left(\begin{array}{l}
\left(\frac{d^{2} x_{1}}{d t^{2}}\right) \\
\left(\begin{array}{l}
d^{2} x_{2} \\
d t^{2}
\end{array}\right) \\
\left(\frac{d^{2} \alpha}{d t^{2}}\right)
\end{array}\right) \\
& \approx\left(m_{3} g\right) \cdot \frac{1}{\left(2 \cdot l_{2}\right)} \\
& +\left(\begin{array}{c}
-x_{1}+x_{2}-l_{1} \alpha \\
-\left(l_{1} \cdot l_{2}+l_{1}^{2}\right) \cdot \alpha-x_{1} \cdot l_{1}+x_{2} \cdot l_{1}
\end{array}\right) \\
& +\left(\begin{array}{c}
F_{1} \\
F_{2} \\
0
\end{array}\right),
\end{aligned}
$$

where $F_{1}=u_{1}(t)[\mathrm{N}]$ and $F_{2}=u_{2}(t)[\mathrm{N}]$ in Figure 6 .

Matrix equation (15) shows that the addition of control into system (15) can be done through the introduction of control forces $F_{1}=u_{1}(t)[\mathrm{N}]$ and $F_{2}=u_{2}(t)[\mathrm{N}]$ in the form of the additional vector components $\left(u_{1}(t) ; u_{2}(t) ; 0\right)^{T}$, added to the right-hand side of equation (15).

Formulation of the governing system of ordinary differential equations (J.4), (J.5), and (J.6) or (15) for motion of the guided mechanical system in Figure 6 allows us to pose the following optimal control problem.

It is necessary to find the control functions $u_{1}(t)$ and $u_{2}(t)$ for control forces $F_{1}=u_{1}(t)[\mathrm{N}]$ and $F_{2}=u_{2}(t)[\mathrm{N}]$ in Figure 6, which provide minimization of the objective function

$$
J=t_{f}+\int_{0}^{t_{f}}(\alpha(t))^{2} d t
$$

where $\alpha(t)[\mathrm{rad}]$ is the variable angle of the cable AE with the vertical axis $z$ and $t_{f}$ [s] is the time of travel of the bridge cranes $A$ and $B$ of the prescribed desired distance $S$.

Minimization of the objective function $J$ (16) has to be done for the following constraints:

$$
\begin{aligned}
& \frac{d}{d t}\left(x_{1}(t)\right)=x_{1 p}(t) \\
& \frac{d}{d t}\left(x_{2}(t)\right)=x_{2 p}(t) ; \\
& \frac{d}{d t}(\alpha(t))=\alpha_{p}(t) ; \\
& \left(m_{1}+m_{3}\right) \cdot\left(\frac{d}{d t}\left(x_{1 p}(t)\right)\right)+m_{3} \cdot l_{1} \cdot\left(\frac{d}{d t}\left(\alpha_{p}(t)\right)\right) \\
& \approx u_{1}(t)-\left(m_{3} g\right) \cdot \frac{1}{\left(2 \cdot l_{2}\right)} \\
& \quad \cdot\left(x_{1}(t)-x_{2}(t)+l_{1} \cdot \alpha(t)\right)
\end{aligned}
$$

$$
\begin{aligned}
& m_{2} \cdot\left(\frac{d}{d t}\left(x_{2 p}(t)\right)\right) \\
& \quad \approx u_{2}(t)+\left(m_{3} g\right) \cdot \frac{1}{\left(2 \cdot l_{2}\right)} \\
& \quad \cdot\left(x_{1}(t)-x_{2}(t)+l_{1} \cdot \alpha(t)\right) ; \\
& m_{3} \cdot l_{1} \cdot\left(\frac{d}{d t}\left(x_{1 p}(t)\right)\right)+m_{3} \cdot l_{1}^{2} \cdot\left(\frac{d}{d t}\left(\alpha_{p}(t)\right)\right) \\
& \quad \approx\left(m_{3} g\right) \cdot \frac{1}{\left(2 \cdot l_{2}\right)} \\
& \quad \cdot\left(-\left(l_{1} \cdot l_{2}+l_{1}^{2}\right) \cdot \alpha(t)-x_{1}(t) \cdot l_{1}+x_{2}(t) \cdot l_{1}\right) ; \\
& x_{1}(0)=0 ; \\
& x_{1 p}(0)=0 ; \\
& x_{2}(0)=0 ; \\
& x_{2}\left(t_{f}\right)=0 ; \\
& x_{2}(t) \mid \leq u_{1 \max } ; \\
& x_{1 p}(t) \mid \leq u_{2 \max } \cdot \\
& x_{2}(0)=0 ; \\
& x_{1}\left(t_{f}\right)=0 ;
\end{aligned}
$$

Introduction of control $\left(u_{1}(t) ; u_{2}(t) ; 0\right)^{T}$ into equations (J.4), (J.5), (J.6), and (15) provides possibility of deriving an optimal control in dependence from the form of a minimized functional $J$ (16) with equal weight coefficients.

\section{Second Computational Example of a Numerical Solution of Derived Governing Equations for the Studied Guided Mechanical System with JModelica.org and Optimica Freeware}

It is possible to achieve a numerical solution of system (16)(25) for minimized functional (16), governing equations (17)(22), initial conditions (23), terminal conditions (24), and limitations on the control forces (25) with module Optimica of freeware code JModelica.org $[14,15,19]$ for the following numerical values of parameters of the mechanical system in 
Figure 6: gravity acceleration is $g=9.81\left(\mathrm{~m} / \mathrm{s}^{2}\right)$; masses of movable carts $A$ and $B$ are $m_{1}=m_{2}=1000(\mathrm{~kg})$; mass of the cross-beam ED is $m_{3}=10000(\mathrm{~kg})$; length of the left rope AE is $l_{1}=6(\mathrm{~m})$; length of the right rope $\mathrm{BD}$ is $l_{2}=5(\mathrm{~m})$; vertical distance between the carts is $b=2(\mathrm{~m})$; length of the crossbeam ED is $a=4(\mathrm{~m})$; prescribed desired transportation distance for bridge cranes $A$ and $B$ is $S=40(\mathrm{~m})$; the maximum value of the first control force is $u_{1 \max }=100(\mathrm{~N})$; and the maximum value of the second control force is $u_{2 \max }=$ $100(\mathrm{~N})$. Derived plots of motion of guided system with zero initial conditions (23) are shown in Figure 7.

\section{Discussion of Derived Results}

The present problem is a new and mathematically complex research problem. Complexity of the problem is connected with the imposition of essentially nonlinear geometric constraints that cause a lot of issues and problems with the derivation of differential equations of motion.

Development of the governing equations was based on the application of a small parameter linearization method. The numerical solution of the problem confirmed the correctness of applying linearization techniques. Derived simulation results were in good agreement with the basic conservation laws of classical mechanics.

The linear momentum of the system at the initial time $t=0$ (s) is determined by the linear momentum of cart $B$ and can be calculated as $m_{2} \cdot V_{B}(0)=1000(\mathrm{~kg}) \cdot 0.5(\mathrm{~m} / \mathrm{s})$ $=500(\mathrm{~kg} \cdot \mathrm{m} / \mathrm{s})$. The total mass of the whole system is $\left(m_{1}+\right.$ $\left.m_{2}+m_{3}\right)=12000(\mathrm{~kg})$. It is possible to determine the linear velocity of the center of gravity of the mechanical system at an arbitrary time with the introduction of a theorem defining center-of-gravity motion conservation as $V_{C}=$ $\left(m_{2} \cdot V_{B}(0)\right) /\left(m_{1}+m_{2}+m_{3}\right)=500(\mathrm{~kg} \cdot \mathrm{m} / \mathrm{s}) / 12000(\mathrm{~kg}) \approx$ $0.042(\mathrm{~m} / \mathrm{s})$. The derived value of $V_{C}$ determines the average velocity for the entire system in Figure 2. So it is possible to estimate the traversed path of the carts $A$ and $B$ in Figure 4(a) for the moment of time $\tau=10$ (s) with the formula $S \approx V_{C}$. $\tau \approx 0.042(\mathrm{~m} / \mathrm{s}) \cdot 10(\mathrm{~s}) \approx 0.42(\mathrm{~m})$. There is good agreement of this estimated value $S \approx 0.42(\mathrm{~m})$ with the numerical value $x_{1 \tau} \approx 0.5(\mathrm{~m})$ in Figure $4(\mathrm{a})$.

It is necessary to note that, in addition to the motion due to the velocity of the center of gravity, there are additional system motions with natural frequencies $k_{1} \approx 1.021(1 / \mathrm{s})$ and $k_{2} \approx 3.794(1 / \mathrm{s})$ (Appendix $\mathrm{H}$ ), and the corresponding natural periods $\tau_{1}=2 \cdot \pi / k_{1} \approx 6.155(\mathrm{~s})$ and $\tau_{2}=2$. $\pi / k_{2} \approx 1.656(\mathrm{~s})$. These two natural periods and amplitudes are clearly observable in Figures 4 and 5.

The numerical solution of an optimal control problem (16)-(25) allows determination of the control variables $u_{1}(t)$ and $u_{2}(t)$ for control forces $F_{1}=u_{1}(t)[\mathrm{N}]$ and $F_{2}=u_{2}(t)[\mathrm{N}]$ in Figure 7(b), which enable transportation of a cross-beam from the initial to the final position with minimum time and minimum swaying in Figures $7(\mathrm{c})$ and $7(\mathrm{~d})$. These control variables $u_{1}(t)$ and $u_{2}(t)$ are the control forces $F_{1}=u_{1}(t)$ and $F_{2}=u_{2}(t)$, applied to the first and second bridge cranes $A$ and $B$. Optimica-derived Figure $7(\mathrm{~b})$ shows that it is possible to provide an optimal motion in the abovementioned sense by the following strategy, when at the first half of motion time the control forces $u_{1}(t)$ and $u_{2}(t)$ should be constant and positive and at the second half of motion time the control forces $u_{1}(t)$ and $u_{2}(t)$ should be negative. As a result, in the beginning, acting forces provide maximum acceleration of the bridge cranes and then deceleration. This control strategy is a "bang-bang" control method with the switching point in the time moment $t^{*}=t_{f} / 2$, where $t_{f}$ is the time of travel of bridge cranes from one position to another. It is shown in Figure 7(b) that during the last 2-3 seconds the control forces have negative values because it is necessary to provide slowing-down (deceleration) of the system motion. In this case the values of control forces are $0.25 \cdot \max \left\{u_{1}(t), u_{2}(t)\right\}$. This kind of a control strategy enables travel to the final position with near zero-values of velocities $V_{x_{1}} \approx V_{x_{2}} \approx V_{x_{3}} \approx$ $0(\mathrm{~m} / \mathrm{s})$ and near zero-values of swaying angles $\alpha \approx \beta \approx 0$. An easy practical realization of these recommendations by bridge cranes operators is the real advantage of the found control strategy. Really, in the first half of the way, crane operators have to accelerate bridge cranes, and in the second half of the movement, crane operators have to decelerate bridge cranes. This control strategy is relatively easy for realization in manual mode using available crane control mechanisms. The proposed control strategy reduces transportation time and partially decreases intensity of the labor of crane operators.

Analysis of Figure 7(a) indicates that the proposed control strategy enables synchronous motion of bridge cranes with quite small and negligible oscillations of a heavy crossbeam. Analysis of Figure 7(c) shows that deviation of a crossbeam from equilibrium position in the horizontal direction does not exceed 0.002 (rad), and in the vertical direction the cross-beam deviation is much smaller and does not exceed $4 \cdot 10^{-6}$ ( $\left.\mathrm{rad}\right)$.

It is easy to confirm the correctness of the proposed optimal control with engineering estimation. According to the law of variation of momentum along the horizontal direction, the impulse of control forces for $F_{1}=u_{1}(t)[\mathrm{N}]$ and $F_{2}=u_{2}(t)[\mathrm{N}]$ is $\left(u_{1}+u_{2}\right) \cdot\left(t_{f} / 2\right) \approx(100+100) \cdot 49(\mathrm{~N} \cdot \mathrm{s})$ $\approx 9800(\mathrm{~N} \cdot \mathrm{s})$. Moreover, it follows from Figure 7(e) that the linear momentum of the system to this time moment $49 \mathrm{~s}$ is $\left(m_{1}+m_{2}+m_{3}\right) \cdot V_{C} \approx(1000+1000+10000) \cdot 0.8(\mathrm{~kg} \cdot \mathrm{m} / \mathrm{s})$ $\approx 9600$ (N.s). The results are approximately the same in the deceleration region. These simple engineering estimations confirm the correctness of the derived optimal control strategy.

Further research directions are associated with dynamic analysis of internal forces, reactions of constraints, introduction of additional elastic-damping devices, development and analysis of other modes of optimal control, introduction of additional elastic-damping devices, and search for other natural frequencies in 3D problem.

\section{Final Conclusions}

The present problem is a truly new problem of lifting-andhandling machinery.

This problem has arisen from applied engineering assignments, associated with civil engineering and transportation problems in plant departments and in field environment conditions. 


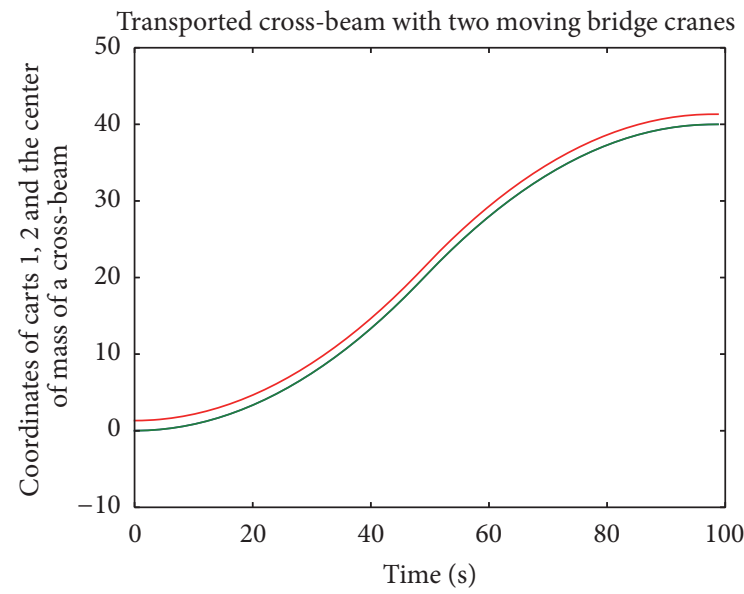

$$
\begin{array}{r}
x_{1} \\
- \\
x_{2}
\end{array}
$$

(a)
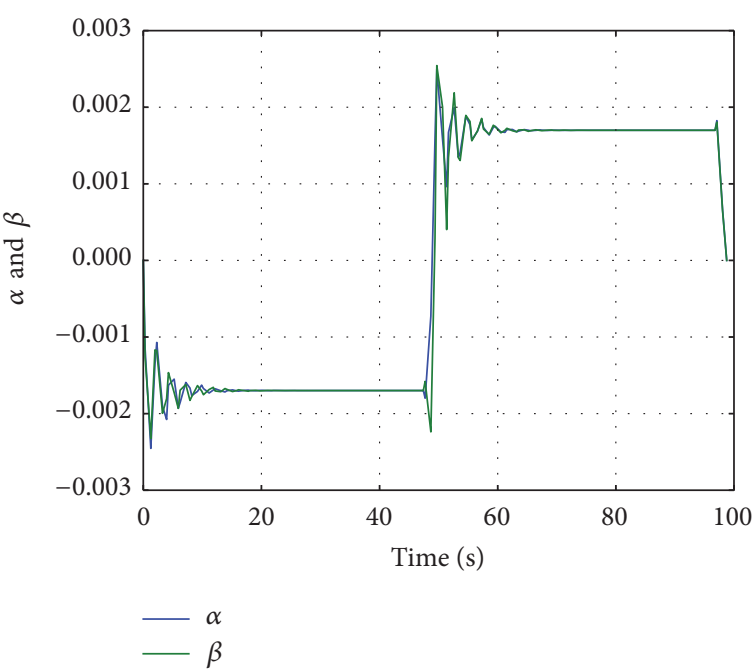

(c)

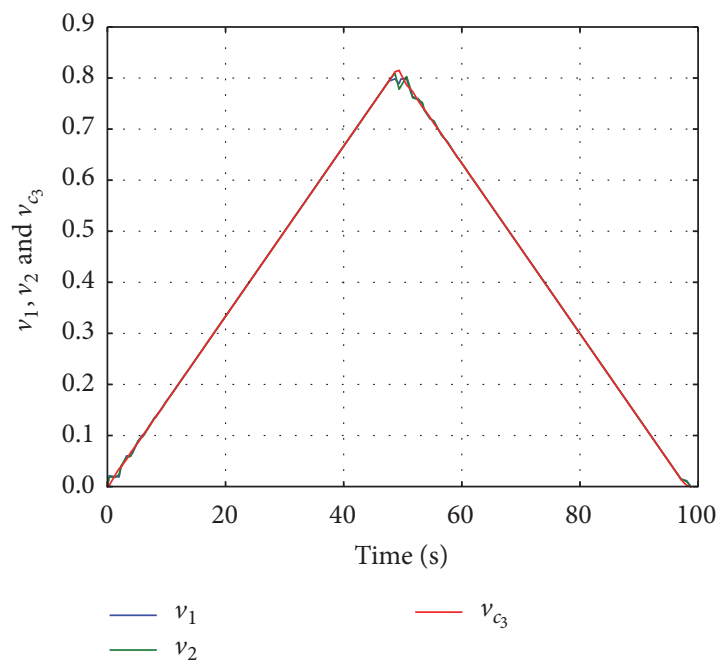

(e)

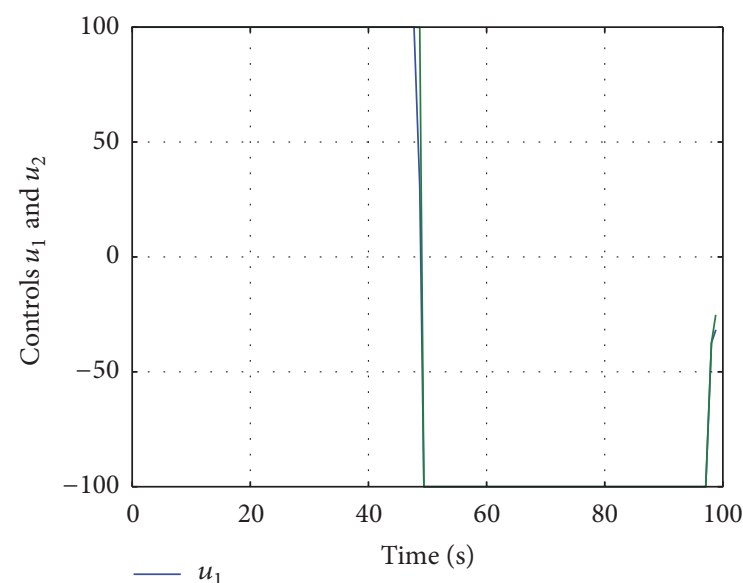

(b)

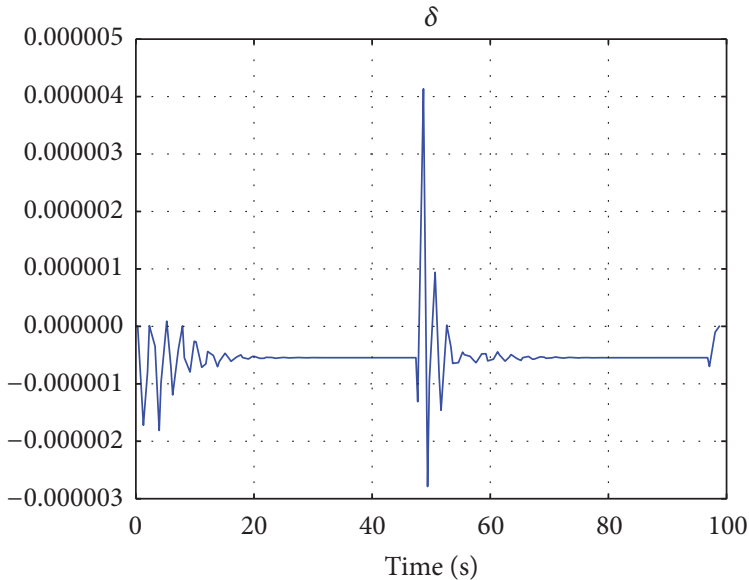

(d)

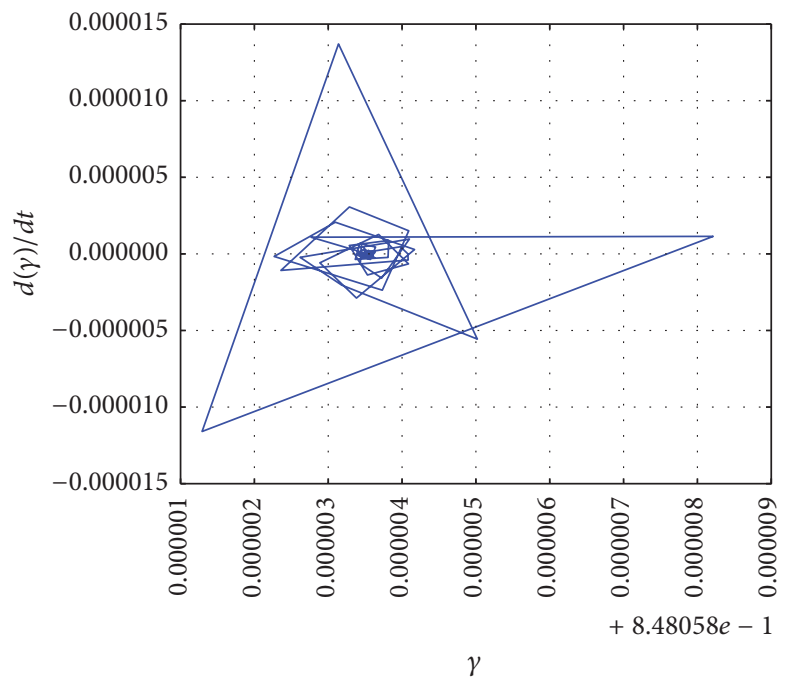

(f)

FIGURE 7: JModelica.org- and Optimica-derived numerical plots for linear coordinates $x_{1}=x_{1}(t) ; x_{2}=x_{2}(t)$; and $x_{3}=x_{3}(t)$ (a); control forces $F_{1}=u_{1}(t)[\mathrm{N}]$ and $F_{2}=u_{2}(t)[\mathrm{N}](\mathrm{b})$; angular coordinates $\alpha=\alpha(t)$ and $\beta=\beta(t)$ (c); angular coordinate $\delta=\delta(t)(\mathrm{d})$; synchronized velocities $V_{x_{1}}(t)=\left(d\left(x_{1}(t)\right) / d t\right), V_{y_{1}}(t)=\left(d\left(y_{1}(t)\right) / d t\right)$, and $V_{x C_{3}}(t)=\left(d\left(x_{C_{3}}(t)\right) / d t\right)$ of points $C_{1}, C_{2}, C_{3}$ during guided system motion $(\mathrm{e})$; and $\omega_{\gamma}=\omega_{\gamma}(\gamma)$; that is, $(d(\gamma(t)) / d t)=f(\gamma)$, obtained for synchronous motion of the mechanical system with an open loop control. 
There are previously known studies of vertical lifting, space canting, and 3D rotation of the load up to $90 \mathrm{deg}$ with two boom cranes, working together. But there is a lack of relevant and specific information concerning horizontal transportation of the load with two bridge cranes.

The present original research article is focused on the development of a mathematical model, describing the horizontal transportation of the load (cross-beam) in the vertical plane.

Transportation dynamics of heavy cross-beam moved by two overhead cranes was addressed with the introduction of the Lagrange equations using small angle assumptions.

A 3DOF mechanical system "heavy cross-beam, two moving carts" was studied.

A system of Lagrange equations for a 3DOF mechanical system was developed and numerically solved.

A number of general trends and features of the heavy cross-beam motion were found using mathematical and numerical simulation techniques. Among motion features are the phenomena of insignificant horizontal swaying of a crossbeam in motion direction as well as infinitesimally small angles of cross-beam oscillations around the center of mass.

One of the methodologies of the search of optimal control was proposed. An optimal control strategy with minimization of transportation time and swaying angles $\alpha$ and $\beta$ was developed with an application of JModelica.org and Optimica freeware.

The proposed mathematical model for an optimal control system allows development of the corresponding graphs and nomographic charts for formulation of applied engineering recommendations.

The posed transport engineering problem will find further development and application with the introduction of elastic-damping devices, changing hoisting cables lengths, and switching to a $3 \mathrm{D}$ simulation.

\section{Appendix}

\section{A. Linearization Techniques}

It is possible to make a linearization of expressions (1) assuming that

$$
\begin{aligned}
& \sin (\alpha) \approx \alpha ; \\
& \cos (\alpha) \approx 1-\left(\frac{1}{2}\right) \cdot \alpha^{2} ; \\
& \sin (\beta) \approx \beta ; \\
& \cos (\beta) \approx 1-\left(\frac{1}{2}\right) \cdot \beta^{2} .
\end{aligned}
$$

However, linearization of $\gamma$ requires the usage of a more sophisticated approach. At the initial moment of time $t=0$ both cables $\mathrm{AE}$ and $\mathrm{BD}$ had the vertical positions with initial values of angles $\alpha_{0}=\beta_{0}=0$ in Figure 3. At $t=0$ the initial inclination angle of the cross-beam $\mathrm{ED}\left(l_{\mathrm{ED}}=a\right)$ to the horizontal is $\gamma_{0}$ (Figure 3). It is obvious that at the initial time moment initial position of oscillating system yields

$$
l_{1}+b=l_{2}+\Delta z_{0} .
$$

The algebraic equation (A.2) yields

$$
\Delta z_{0}=l_{1}-l_{2}+b .
$$

Equations (A.2) and (A.3) enable calculation of the initial angle $\gamma_{0}$ :

$$
\begin{aligned}
& \sin \left(\gamma_{0}\right)=\frac{\Delta z_{0}}{a}=\frac{\left(l_{1}-l_{2}+b\right)}{a} ; \\
& \cos \left(\gamma_{0}\right)=\sqrt{1-\sin ^{2}\left(\gamma_{0}\right)} .
\end{aligned}
$$

Further linearization of (1) requires the derivation of simplified trigonometric expressions for the current angle $\gamma$ using the formulae for sine and cosine of angular sum, where $\gamma=\gamma_{0}+\delta$ (Figure 2):

$$
\begin{aligned}
\cos (\gamma) & =\cos \left(\gamma_{0}+\delta\right) \\
& =\cos \left(\gamma_{0}\right) \cos (\delta)-\sin \left(\gamma_{0}\right) \sin (\delta) ; \\
\sin (\gamma) & =\sin \left(\gamma_{0}+\delta\right) \\
& =\sin \left(\gamma_{0}\right) \cos (\delta)+\cos \left(\gamma_{0}\right) \sin (\delta) .
\end{aligned}
$$

It is possible to make a linearization of (A.5) for the small angle $\delta$ assuming that

$$
\begin{aligned}
& \sin (\delta) \approx \delta ; \\
& \cos (\delta) \approx 1 .
\end{aligned}
$$

After substitution of (A.6) into (A.5) one obtains

$$
\begin{aligned}
& \cos (\gamma)=\cos \left(\gamma_{0}+\delta\right) \approx \cos \left(\gamma_{0}\right)-\delta \cdot \sin \left(\gamma_{0}\right) ; \\
& \sin (\gamma)=\sin \left(\gamma_{0}+\delta\right) \approx \sin \left(\gamma_{0}\right)+\delta \cdot \cos \left(\gamma_{0}\right) .
\end{aligned}
$$

As will subsequently be shown in further equations (4), (B.9), and (5), the angle $\delta$ and the angular velocity of the cross-beam ED are the second-order infinitesimal quantities with respect to $\alpha$; that is,

$$
\begin{aligned}
\delta & =O\left(\alpha^{2}\right) ; \\
\left(\frac{d \delta}{d t}\right) & =\left(\frac{d \gamma}{d t}\right)=O\left(\alpha^{2}\right) .
\end{aligned}
$$

Substitution of (A.9) into (A.7) approximately results in

$$
\cos (\gamma) \approx \cos \left(\gamma_{0}\right) \text {. }
$$

Combined usage of (A.1) and (A.10) provides a way to linearize (1) for the abscissa $x_{B}$ of point $B$. Substitution of (A.1) and (A.10) into the upper lines of (1) yields that

$$
\begin{aligned}
x_{B} & =a \cos \left(\gamma_{0}\right)+x_{2} \\
& \approx x_{1}+l_{1} \cdot \alpha+a \cos \left(\gamma_{0}\right)-l_{2} \cdot \beta .
\end{aligned}
$$

Approximate equation (A.11) results in

$$
x_{2}-x_{1} \approx l_{1} \cdot \alpha-l_{2} \cdot \beta .
$$




\section{B. Estimation of Small Inclination Angles}

Now it is necessary to find a simplified expression for $\gamma=$ $f_{\gamma}\left(x_{1}, x_{2}, \alpha\right)$, where $\gamma=\gamma_{0}+\delta$. For this purpose it is necessary to address system (1) for the applicate $z_{B}$ of point $B$. Substitution of the second equations of system (A.1) and (A.8) into the lower lines of (1) yields

$$
\begin{aligned}
z_{B}= & b \\
\approx & -l_{1} \cdot\left(1-\left(\frac{1}{2}\right) \cdot \alpha^{2}\right)+a \cdot \sin \left(\gamma_{0}\right)+a \cdot \delta \\
& \cdot \cos \left(\gamma_{0}\right)+l_{2} \cdot\left(1-\left(\frac{1}{2}\right) \cdot \beta^{2}\right),
\end{aligned}
$$

where the second term on the right-hand side of (B.1) is determined by expression (A.4). Therefore, the substitution of (A.4) into (B.1) produces

$$
\begin{aligned}
b \approx & -l_{1} \cdot\left(1-\left(\frac{1}{2}\right) \cdot \alpha^{2}\right)+\left(l_{1}-l_{2}+b\right)+a \cdot \delta \\
& \cdot \cos \left(\gamma_{0}\right)+l_{2} \cdot\left(1-\left(\frac{1}{2}\right) \cdot \beta^{2}\right) \\
b \approx & -l_{1}+\left(\frac{1}{2}\right) \cdot l_{1} \cdot \alpha^{2}+l_{1}-l_{2}+b+a \cdot \delta \cdot \cos \left(\gamma_{0}\right) \\
& +l_{2}-\left(\frac{1}{2}\right) \cdot l_{2} \cdot \beta^{2} .
\end{aligned}
$$

It is obvious that components $b, l_{1}$, and $l_{2}$ with opposite signs in (B.3) result in zero-sum terms of (B.3). So (B.3) yields

$$
a \cdot \delta \cdot \cos \left(\gamma_{0}\right) \approx\left(\frac{1}{2}\right) \cdot l_{2} \cdot \beta^{2}-\left(\frac{1}{2}\right) \cdot l_{1} \cdot \alpha^{2} .
$$

After transformation (B.4) yields the small angle $\delta$ expression:

$$
\delta \approx \frac{\left(l_{2} \cdot \beta^{2}-l_{1} \cdot \alpha^{2}\right)}{\left(2 \cdot a \cdot \cos \left(\gamma_{0}\right)\right)} .
$$

Equation (B.5) determines the small inclination angle $\delta$ as the simplified linear function of the variables $\alpha$ and $\beta$; that is, $\delta=f_{\delta}(\alpha, \beta)$. Equation (B.5) provides the possibility of calculating an approximate expression for the small angle $\gamma$ for cross-beam ED inclination in Figure 2:

$$
\gamma=\gamma_{0}+\delta \approx \gamma_{0}+\left(\frac{\left(l_{2} \cdot \beta^{2}-l_{1} \cdot \alpha^{2}\right)}{\left(2 \cdot a \cdot \cos \left(\gamma_{0}\right)\right)}\right) .
$$

The derived equations (B.5) and (B.6) provide a way to estimate the value of the angular velocity $\omega_{\delta}=(d \delta / d t)=$ $\omega_{\gamma}=(d \gamma / d t)$ by the following expression:

$$
\begin{aligned}
& \left(\frac{d \gamma}{d t}\right)=\left(\frac{d \delta}{d t}\right) \\
& \quad \approx \frac{1}{\left(a \cdot \cos \left(\gamma_{0}\right)\right)}\left(l_{2} \cdot \beta \cdot\left(\frac{d \beta}{d t}\right)-l_{1} \cdot \alpha \cdot\left(\frac{d \alpha}{d t}\right)\right) .
\end{aligned}
$$

It is possible to make further transformations of (B.5) and to derive the small inclination angle $\delta$ as the simplified linear function of the variables $x_{1}, x_{2}, \alpha$, that is, to get an expression for $\delta=f_{\delta}\left(x_{1}, x_{2}, \alpha\right)$ by substitution of (2) into (B.5):

$$
\begin{aligned}
\delta & \approx \frac{1}{\left(2 \cdot a \cdot \cos \left(\gamma_{0}\right)\right)}\left(l_{2} \cdot\left(\frac{1}{l_{2}}\right)^{2} \cdot\left(x_{1}-x_{2}+l_{1} \alpha\right)^{2}\right. \\
& \left.-l_{1} \cdot \alpha^{2}\right) .
\end{aligned}
$$
yields

Reducing expression (B.8) to a common denominator

$$
\begin{aligned}
& \delta \approx \\
& \qquad \frac{1}{\left(2 \cdot a \cdot l_{2} \cdot \cos \left(\gamma_{0}\right)\right)}\left(\left(x_{1}-x_{2}+l_{1} \alpha\right)^{2}-l_{1} \cdot l_{2}\right. \\
& \left.\cdot \alpha^{2}\right) .
\end{aligned}
$$

\section{Linearized Coordinates of Cross-Beam Center}

Now it is necessary to address the coordinates and displacements of the point $C_{3}$, which is located at the center of the cross-beam ED. The coordinates of the point $C_{3}$ are necessary for determination of kinetic (Appendix F) and potential energies of the cross-beam ED, as well as for calculation of the generalized forces (Appendix E) in the Lagrange equations (Appendix G). The horizontal and vertical Cartesian coordinates of point $C_{3}$ in Figure 2 are determined by the following formulae:

$$
\begin{aligned}
& x_{C_{3}}=x_{1}+l_{1} \cdot \sin (\alpha)+\left(\frac{a}{2}\right) \cdot \cos (\gamma) ; \\
& z_{C_{3}}=-l_{1} \cdot \cos (\alpha)+\left(\frac{a}{2}\right) \cdot \sin (\gamma) .
\end{aligned}
$$

Further linearization of (C.1) is possible through the application of the previous expressions (A.1) and (A.7)-(A.8). Substitution of (A.1) and (A.7)-(A.8) yields

$$
\begin{aligned}
x_{C_{3}} \approx & x_{1}+l_{1} \cdot \alpha+\left(\frac{a}{2}\right) \cdot\left(\cos \left(\gamma_{0}\right)-\delta \cdot \sin \left(\gamma_{0}\right)\right) ; \\
z_{C_{3}} \approx & -l_{1} \cdot\left(1-\left(\frac{1}{2}\right) \cdot \alpha^{2}\right)+\left(\frac{a}{2}\right) \\
& \cdot\left(\sin \left(\gamma_{0}\right)+\delta \cdot \cos \left(\gamma_{0}\right)\right) .
\end{aligned}
$$

It is possible to expand the brackets in (C.2) to get the following simplified expressions:

$$
\begin{aligned}
x_{C_{3}} \approx & x_{1}+l_{1} \cdot \alpha-\left(\left(\frac{a}{2}\right) \cdot \sin \left(\gamma_{0}\right)\right) \cdot \delta \\
& +\left(\left(\frac{a}{2}\right) \cdot \cos \left(\gamma_{0}\right)\right) ; \\
z_{C_{3}} \approx & \left(\left(\frac{1}{2}\right) \cdot l_{1}\right) \cdot \alpha^{2}+\left(\left(\frac{a}{2}\right) \cdot \cos \left(\gamma_{0}\right)\right) \cdot \delta \\
& +\left(\left(\frac{a}{2}\right) \cdot \sin \left(\gamma_{0}\right)-l_{1}\right) .
\end{aligned}
$$


Substitution of (A.4) and (B.9) into the second equation of system (C.3) results in the following expression for the vertical location applicate of the point $C_{3}$ :

$$
\begin{aligned}
& z_{C_{3}} \approx\left(\left(\frac{1}{2}\right) \cdot l_{1}\right) \cdot \alpha^{2}+\left(\left(\frac{a}{2}\right) \cdot \cos \left(\gamma_{0}\right)\right) \\
& \cdot \frac{1}{\left(2 \cdot a \cdot l_{2} \cdot \cos \left(\gamma_{0}\right)\right)}\left(\left(x_{1}-x_{2}+l_{1} \alpha\right)^{2}-l_{1} \cdot l_{2}\right. \\
&\left.\cdot \alpha^{2}\right)+\left(\left(\frac{1}{2}\right) \cdot\left(l_{1}-l_{2}+b\right)-l_{1}\right) .
\end{aligned}
$$

\section{Linearized Velocities of Cross-Beam Center}

The further calculation of kinetic energy of the cross-beam ED requires an estimation of velocity of the point $C_{3}$. The first time derivatives of system (C.1) give to one the system of equations for $\overrightarrow{\mathbf{V}}_{\mathrm{C}_{3}}$ projections:

$$
\begin{aligned}
\frac{d\left(x_{C_{3}}\right)}{d t}= & \left(\frac{d x_{1}}{d t}\right)+\left(l_{1} \cdot \cos (\alpha)\right) \cdot\left(\frac{d \alpha}{d t}\right) \\
& -\left(\left(\frac{a}{2}\right) \cdot \sin (\gamma)\right) \cdot\left(\frac{d \gamma}{d t}\right) ; \\
\frac{d\left(z_{C_{3}}\right)}{d t}= & \left(l_{1} \cdot \sin (\alpha)\right) \cdot\left(\frac{d \alpha}{d t}\right)+\left(\left(\frac{a}{2}\right) \cdot \cos (\gamma)\right) \\
& \cdot\left(\frac{d \gamma}{d t}\right) .
\end{aligned}
$$

It is more useful to linearize system (D.1) through differentiation of system (C.3) with respect to the time $t$ :

$$
\begin{aligned}
\frac{d\left(x_{C_{3}}\right)}{d t} \approx & \left(\frac{d x_{1}}{d t}\right)+l_{1} \cdot\left(\frac{d \alpha}{d t}\right)-\left(\left(\frac{a}{2}\right) \cdot \sin \left(\gamma_{0}\right)\right) \\
& \cdot\left(\frac{d \delta}{d t}\right) ; \\
\frac{d\left(z_{C_{3}}\right)}{d t} \approx & \left(\left(\frac{1}{2}\right) \cdot l_{1}\right) \cdot(2 \cdot \alpha) \cdot\left(\frac{d \alpha}{d t}\right) \\
& +\left(\left(\frac{a}{2}\right) \cdot \cos \left(\gamma_{0}\right)\right) \cdot\left(\frac{d \delta}{d t}\right) .
\end{aligned}
$$

The system of expressions (D.2) can be further simplified by taking into account equation (5) that $\omega_{3}=\omega_{\delta}=(d \delta / d t)=$ $\omega_{\gamma}=(d \gamma / d t)=O\left(\alpha^{2}\right) \approx 0$ :

$$
\begin{aligned}
& \frac{d\left(x_{C_{3}}\right)}{d t} \approx\left(\frac{d x_{1}}{d t}\right)+l_{1} \cdot\left(\frac{d \alpha}{d t}\right) ; \\
& \frac{d\left(z_{C_{3}}\right)}{d t} \approx\left(l_{1} \cdot \alpha\right) \cdot\left(\frac{d \alpha}{d t}\right) .
\end{aligned}
$$

It is possible to further simplify (D.3) by taking into account the fact that the product $\alpha \cdot(d \alpha / d t)$ is the secondorder infinitesimal quantity; that is,

$$
\alpha \cdot\left(\frac{d \alpha}{d t}\right)=O\left(\alpha^{2}\right)
$$

Substitution of (D.4) into (D.3) yields

$$
\begin{aligned}
& \frac{d\left(x_{C_{3}}\right)}{d t} \approx\left(\frac{d x_{1}}{d t}\right)+l_{1} \cdot\left(\frac{d \alpha}{d t}\right) \\
& \frac{d\left(z_{C_{3}}\right)}{d t} \approx 0 .
\end{aligned}
$$

\section{E. Generalized Forces}

The first generalized force $Q_{x_{1}}$, which corresponds to the generalized linear coordinate $x_{1}$, is as follows:

$$
\begin{aligned}
Q_{x_{1}} & =-\frac{\partial \Pi}{\partial x_{1}} \\
& \approx(-1) \cdot\left(m_{3} g\right) \cdot \frac{1}{\left(4 \cdot l_{2}\right)} \cdot 2 \cdot\left(x_{1}-x_{2}+l_{1} \alpha\right) .
\end{aligned}
$$

After simplifications the previous equation (E.1) for the first generalized force $Q_{x_{1}}$ takes the form

$$
Q_{x_{1}} \approx(-1) \cdot\left(m_{3} g\right) \cdot \frac{1}{\left(2 \cdot l_{2}\right)} \cdot\left(x_{1}-x_{2}+l_{1} \alpha\right) \text {. }
$$

The second generalized force $Q_{x_{2}}$, which corresponds to the generalized linear coordinate $x_{2}$, can be calculated as

$$
\begin{aligned}
Q_{x_{2}}= & -\frac{\partial \Pi}{\partial x_{2}} \\
\approx & (-1) \cdot\left(m_{3} g\right) \cdot \frac{1}{\left(4 \cdot l_{2}\right)} \cdot 2 \cdot\left(x_{1}-x_{2}+l_{1} \alpha\right) \\
& \cdot(-1) .
\end{aligned}
$$

After simplifications (E.3) for the second generalized force $Q_{x_{2}}$ yields

$$
Q_{x_{2}} \approx(+1) \cdot\left(m_{3} g\right) \cdot \frac{1}{\left(2 \cdot l_{2}\right)} \cdot\left(x_{1}-x_{2}+l_{1} \alpha\right) \text {. }
$$

It is possible to calculate the third generalized force $Q_{\alpha}$, which corresponds to the generalized angular coordinate $\alpha$, by the following expression:

$$
\begin{aligned}
Q_{\alpha} & =-\frac{\partial \Pi}{\partial \alpha} \approx(-1) \cdot\left(m_{3} g\right) \cdot\left(\left(\left(\frac{1}{2}\right) \cdot l_{1}\right) \cdot(2 \cdot \alpha)\right. \\
& \left.+\frac{1}{\left(4 \cdot l_{2}\right)}\left(2 \cdot\left(x_{1}-x_{2}+l_{1} \alpha\right) \cdot l_{1}-l_{1} \cdot l_{2} \cdot 2 \cdot \alpha\right)\right) .
\end{aligned}
$$


After simplifications (E.5) produces

$$
\begin{aligned}
Q_{\alpha} & \approx(-1) \cdot\left(m_{3} g\right) \cdot\left(\left(l_{1} \cdot \alpha\right)\right. \\
& \left.+\frac{1}{\left(2 \cdot l_{2}\right)}\left(\left(x_{1}-x_{2}+l_{1} \alpha\right) \cdot l_{1}-l_{1} \cdot l_{2} \cdot \alpha\right)\right) .
\end{aligned}
$$

Reducing a right-hand side fraction of (E.6) to a common denominator yields

$$
\begin{aligned}
Q_{\alpha} \approx & \left(m_{3} g\right) \cdot \frac{1}{\left(2 \cdot l_{2}\right)} \\
& \cdot\left(-l_{1} \cdot l_{2} \cdot \alpha-\left(x_{1}-x_{2}+l_{1} \alpha\right) \cdot l_{1}\right) .
\end{aligned}
$$

Expanding the brackets in (E.7) leads to the following expression:

$$
\begin{aligned}
Q_{\alpha} \approx & \left(m_{3} g\right) \cdot \frac{1}{\left(2 \cdot l_{2}\right)} \\
& \cdot\left(-l_{1} \cdot l_{2} \cdot \alpha-x_{1} \cdot l_{1}+x_{2} \cdot l_{1}-l_{1}^{2} \cdot \alpha\right) .
\end{aligned}
$$

Equation (E.8) finally produces

$$
\begin{aligned}
Q_{\alpha} \approx & \left(m_{3} g\right) \cdot \frac{1}{\left(2 \cdot l_{2}\right)} \\
& \cdot\left(-\left(l_{1} \cdot l_{2}+l_{1}^{2}\right) \cdot \alpha-x_{1} \cdot l_{1}+x_{2} \cdot l_{1}\right) .
\end{aligned}
$$

\section{F. System Kinetic Energy}

Calculation of kinetic energy of the mechanical system in Figure 2 requires some additional comments.

It is obvious that the first cart $A$ (point $C_{1}$ ) is involved in the translational motion along the horizontal axis $O x$. Hence, the kinetic energy of a 2D model of the bridge crane $A$ can be calculated by the following expression:

$$
\begin{aligned}
T_{1} & =\left(\frac{1}{2}\right) \cdot m_{A} \cdot\left(\overrightarrow{\mathbf{V}}_{C_{1}}\right)^{2}=\left(\frac{1}{2}\right) \cdot m_{A} \cdot\left(V_{C_{1}}\right)^{2} \\
& =\left(\frac{1}{2}\right) \cdot m_{1} \cdot\left(\frac{d x_{1}}{d t}\right)^{2} .
\end{aligned}
$$

It is clearly shown in Figure 2 that the second cart $B$ (point $C_{2}$ ) is involved in the translational motion along the upper horizontal axis, which is parallel to axis $O x$ and is located from $O x$ at the distance $b$. Therefore, the kinetic energy of a $2 \mathrm{D}$ model of the bridge crane $B$ can be analogously calculated by the same formula for kinetic energy of translational motion as

$$
\begin{aligned}
T_{2} & =\left(\frac{1}{2}\right) \cdot m_{B} \cdot\left(\overrightarrow{\mathbf{V}}_{C_{2}}\right)^{2}=\left(\frac{1}{2}\right) \cdot m_{B} \cdot\left(V_{C_{2}}\right)^{2} \\
& =\left(\frac{1}{2}\right) \cdot m_{2} \cdot\left(\frac{d x_{2}}{d t}\right)^{2} .
\end{aligned}
$$

It is shown in Figures 1 and 2 that the heavy cross-beam ED can be considered as a solid rod, which is involved in parallel-plane motion. It is well known from the course of mechanics that the kinetic energy of the cross-beam ED in parallel-plane motion can be calculated with König's theorem as

$$
T_{3}=\left(\frac{1}{2}\right) \cdot m_{\mathrm{ED}} \cdot\left(\overrightarrow{\mathbf{V}}_{\mathrm{C}_{3}}\right)^{2}+\left(\frac{1}{2}\right) \cdot J_{\mathrm{C}_{3}} \cdot\left(\overrightarrow{\boldsymbol{\omega}}_{\mathrm{ED}}\right)^{2},
$$

where $m_{\mathrm{ED}}=m_{3}$ is the mass of the cross-beam ED, $\mathbf{V}_{C_{3}}=$ $\mathbf{V}_{C_{3} x}+\mathbf{V}_{C_{3} z}$ is the vector of the linear velocity of the point $C_{3}$ (see formulae (D.1)-(D.5) of Appendix D), where point $C_{3}$ is the center of mass of the cross-beam ED, $J_{C_{3}}=((1 / 12)$. $\left.m_{3} \cdot a^{2}\right)$ is the moment of inertia of the cross-beam ED with respect to the cross-beam central point $C_{3}$, and $\boldsymbol{\omega}_{\mathrm{ED}}=\boldsymbol{\omega}_{3}$ is the vector of an angular velocity of the cross-beam ED, which is determined by formulae (B.7) and (5).

Substitution of scalar quantities into König's theorem results in the following equation:

$$
\begin{aligned}
T_{3}= & \left(\frac{1}{2}\right) \cdot m_{3} \cdot\left(\left(\frac{d\left(x_{C_{3}}\right)}{d t}\right)^{2}+\left(\frac{d\left(z_{C_{3}}\right)}{d t}\right)^{2}\right) \\
& +\left(\frac{1}{2}\right) \cdot\left(\frac{1}{12}\left(m_{3} \cdot a^{2}\right)\right) \cdot\left(\frac{d \gamma}{d t}\right)^{2},
\end{aligned}
$$

where approximate values of time derivatives $\left(d\left(x_{C_{3}}\right) / d t\right)$, $\left(d\left(z_{C_{3}}\right) / d t\right)$, and $(d \gamma / d t)$ are determined by (5) and (D.5).

It was shown above that the first time derivatives $\left(d\left(z_{C_{3}}\right) / d t\right)$ and $(d \gamma / d t)$ are second-order infinitesimal quantities. Therefore substitution of (5) and (D.5) into (F.4) results in zero-values of the following two terms of (F.4): $\left(d\left(z_{\mathrm{C}_{3}}\right) / d t\right)^{2} \approx 0$ and $(d \gamma / d t)^{2} \approx 0$. Substitution of (5) and (D.5) into (F.4) proves the possibility of reducing the parallelplane motion of the heavy cross-beam ED to planar motion.

Simplified formula (F.4) after linearization with (5) and (D.5) yields that

$$
T_{3} \approx\left(\frac{1}{2}\right) \cdot m_{3} \cdot\left(\frac{d\left(x_{C_{3}}\right)}{d t}\right)^{2} .
$$

Substitution of (D.5) into (F.5) results in

$$
T_{3} \approx\left(\frac{1}{2}\right) \cdot m_{3} \cdot\left(\left(\frac{d x_{1}}{d t}\right)+l_{1} \cdot\left(\frac{d \alpha}{d t}\right)\right)^{2} .
$$

It is possible to calculate the kinetic energy of mechanical system by substitution of (F.1), (F.2), and (F.6) into algebraic expression for the total kinetic energy:

$$
\begin{aligned}
T= & T_{1}+T_{2}+T_{3} \\
\approx & \left(\frac{1}{2}\right) \cdot m_{1} \cdot\left(\frac{d x_{1}}{d t}\right)^{2}+\left(\frac{1}{2}\right) \cdot m_{2} \cdot\left(\frac{d x_{2}}{d t}\right)^{2} \\
& +\left(\frac{1}{2}\right) \cdot m_{3} \cdot\left(\frac{d x_{1}}{d t}\right)^{2}+\left(\frac{1}{2}\right) \cdot m_{3} \cdot l_{1}^{2} \cdot\left(\frac{d \alpha}{d t}\right)^{2} \\
& +\left(\frac{1}{2}\right) \cdot m_{3} \cdot 2 \cdot\left(\frac{d x_{1}}{d t}\right) \cdot l_{1} \cdot\left(\frac{d \alpha}{d t}\right) .
\end{aligned}
$$




\section{G. System Lagrange Equations}

The first Lagrange equation for the generalized coordinate $x_{1}$ is as follows:

$$
\frac{d}{d t}\left(\frac{\partial T}{\partial \dot{x}_{1}}\right)-\frac{\partial T}{\partial x_{1}}=Q_{x_{1}}
$$

where $T$ and $Q_{x_{1}}$ are approximately determined by (10) and (E.2).

The partial derivative of the kinetic energy with respect to the first generalized velocity $\left(d x_{1} / d t\right)$ yields

$$
\begin{aligned}
\frac{\partial T}{\partial \dot{x}_{1}} \approx & \left(\frac{1}{2}\right) \cdot\left(m_{1}+m_{3}\right) \cdot 2 \cdot\left(\frac{d x_{1}}{d t}\right)+m_{3} \cdot l_{1} \\
& \cdot\left(\frac{d \alpha}{d t}\right) .
\end{aligned}
$$

The full derivative of expression (G.2) with respect to time $t$ is as follows:

$$
\begin{gathered}
\frac{d}{d t}\left(\frac{\partial T}{\partial \dot{x}_{1}}\right) \approx \\
\left(m_{1}+m_{3}\right) \cdot\left(\frac{d^{2} x_{1}}{d t^{2}}\right)+m_{3} \cdot l_{1} \\
\cdot\left(\frac{d^{2} \alpha}{d t^{2}}\right) .
\end{gathered}
$$

It follows from (10) that the partial derivative of the kinetic energy with respect to the first generalized coordinate $x_{1}$ has a zero-value

$$
\frac{\partial T}{\partial x_{1}} \approx 0
$$

Substitution of (G.3) and (G.4) into (G.1) results in the following approximate expression of the left-hand side of the first Lagrange equation:

$$
\begin{gathered}
\frac{d}{d t}\left(\frac{\partial T}{\partial \dot{x}_{1}}\right)-\frac{\partial T}{\partial x_{1}} \approx \\
\left(m_{1}+m_{3}\right) \cdot\left(\frac{d^{2} x_{1}}{d t^{2}}\right)+m_{3} \cdot l_{1} \\
\cdot\left(\frac{d^{2} \alpha}{d t^{2}}\right) .
\end{gathered}
$$

Substitution of (G.5) and (E.2) into (G.1) gives the first simplified Lagrange equation:

$$
\begin{aligned}
& \left(m_{1}+m_{3}\right) \cdot\left(\frac{d^{2} x_{1}}{d t^{2}}\right)+m_{3} \cdot l_{1} \cdot\left(\frac{d^{2} \alpha}{d t^{2}}\right) \\
& \approx(-1) \cdot\left(m_{3} g\right) \cdot \frac{1}{\left(2 \cdot l_{2}\right)} \cdot\left(x_{1}-x_{2}+l_{1} \alpha\right) .
\end{aligned}
$$

The second Lagrange equation for the generalized coordinate $x_{2}$ is as follows:

$$
\frac{d}{d t}\left(\frac{\partial T}{\partial \dot{x}_{2}}\right)-\frac{\partial T}{\partial x_{2}}=Q_{x_{2}}
$$

where $T$ and $Q_{x_{2}}$ are approximately determined by (10) and (E.4).

The partial derivative of the kinetic energy with respect to the second generalized velocity $\left(d x_{2} / d t\right)$ yields

$$
\frac{\partial T}{\partial \dot{x}_{2}} \approx\left(\frac{1}{2}\right) \cdot m_{2} \cdot 2 \cdot\left(\frac{d x_{2}}{d t}\right)
$$

The full derivative of expression (G.8) with respect to time $t$ is as follows:

$$
\frac{d}{d t}\left(\frac{\partial T}{\partial \dot{x}_{2}}\right) \approx m_{2} \cdot\left(\frac{d^{2} x_{2}}{d t^{2}}\right)
$$

It follows from (10) that the partial derivative of the kinetic energy with respect to the second generalized coordinate $x_{2}$ has a zero-value

$$
\frac{\partial T}{\partial x_{2}} \approx 0
$$

Substitution of (G.9) and (G.10) into (G.7) results in the following approximate expression of the left-hand side of the second Lagrange equation:

$$
\frac{d}{d t}\left(\frac{\partial T}{\partial \dot{x}_{2}}\right)-\frac{\partial T}{\partial x_{2}} \approx m_{2} \cdot\left(\frac{d^{2} x_{2}}{d t^{2}}\right)
$$

Substitution of (G.11) and (E.4) into (G.7) gives the second simplified Lagrange equation:

$$
\begin{aligned}
m_{2} \cdot\left(\frac{d^{2} x_{2}}{d t^{2}}\right) \approx & (+1) \cdot\left(m_{3} g\right) \cdot \frac{1}{\left(2 \cdot l_{2}\right)} \\
& \cdot\left(x_{1}-x_{2}+l_{1} \alpha\right) .
\end{aligned}
$$

The third Lagrange equation for the generalized coordinate $\alpha$ is as follows:

$$
\frac{d}{d t}\left(\frac{\partial T}{\partial \dot{\alpha}}\right)-\frac{\partial T}{\partial \alpha}=Q_{\alpha}
$$

where $T$ and $Q_{\alpha}$ are approximately determined by (10) and (E.9).

The partial derivative of the kinetic energy with respect to the third generalized velocity $(d \alpha / d t)$ yields

$$
\frac{\partial T}{\partial \dot{\alpha}} \approx\left(\frac{1}{2}\right) \cdot m_{3} \cdot l_{1}^{2} \cdot 2 \cdot\left(\frac{d \alpha}{d t}\right)+m_{3} \cdot l_{1} \cdot\left(\frac{d x_{1}}{d t}\right) .
$$

The full derivative of expression (G.14) with respect to time $t$ is as follows:

$$
\frac{d}{d t}\left(\frac{\partial T}{\partial \dot{\alpha}}\right) \approx m_{3} \cdot l_{1}^{2} \cdot\left(\frac{d^{2} \alpha}{d t^{2}}\right)+m_{3} \cdot l_{1} \cdot\left(\frac{d^{2} x_{1}}{d t^{2}}\right)
$$


It follows from (10) that the partial derivative of the kinetic energy with respect to the third generalized coordinate $\alpha$ has a zero-value

$$
\frac{\partial T}{\partial \alpha} \approx 0
$$

Substitution of (G.15) and (G.16) into (G.13) results in the following approximate expression of the left-hand side of the third Lagrange equation:

$$
\begin{gathered}
\frac{d}{d t}\left(\frac{\partial T}{\partial \dot{\alpha}}\right)-\frac{\partial T}{\partial \alpha} \approx m_{3} \cdot l_{1}^{2} \cdot\left(\frac{d^{2} \alpha}{d t^{2}}\right)+m_{3} \cdot l_{1} \\
\cdot\left(\frac{d^{2} x_{1}}{d t^{2}}\right) .
\end{gathered}
$$

Substitution of (G.17) and (E.9) into (G.13) gives the third simplified Lagrange equation:

$$
\begin{aligned}
m_{3} & \cdot l_{1}^{2} \cdot\left(\frac{d^{2} \alpha}{d t^{2}}\right)+m_{3} \cdot l_{1} \cdot\left(\frac{d^{2} x_{1}}{d t^{2}}\right) \\
\approx & \left(m_{3} g\right) \cdot \frac{1}{\left(2 \cdot l_{2}\right)} \\
& \cdot\left(-\left(l_{1} \cdot l_{2}+l_{1}^{2}\right) \cdot \alpha-x_{1} \cdot l_{1}+x_{2} \cdot l_{1}\right) .
\end{aligned}
$$

\section{H. Numerical Estimation of Natural Frequencies for the Mechanical System}

It is possible to estimate the natural frequencies of the mechanical system with the system (13) of Lagrange equations (G.6), (G.12), and (G.18). After generation of the determinant of the natural frequencies matrix for system (13) it is possible to write the following characteristic biquadratic equation for system (13) of Lagrange equations (G.6), (G.12), and (G.18) in the form:

$$
\left|\begin{array}{ccc}
\left(\frac{\left(m_{1}+m_{3}\right)}{m_{3}} \cdot \lambda^{2}+\frac{g}{2 \cdot l_{2}}\right) & \left(-\frac{g}{2 \cdot l_{2}}\right) & \left(\lambda^{2}+\frac{g}{2 \cdot l_{2}}\right) \\
\left(-\frac{g}{2 \cdot l_{2}}\right) & \left(\frac{m_{2}}{m_{3}} \cdot \lambda^{2}+\frac{g}{2 \cdot l_{2}}\right) & \left(-\frac{g \cdot l_{1}}{2 \cdot l_{2}}\right) \\
\left(\lambda^{2}+\frac{g}{2 \cdot l_{2}}\right) & \left(-\frac{g \cdot l_{1}}{2 \cdot l_{2}}\right) & \left(l_{1} \cdot \lambda^{2}+\frac{g}{2 \cdot l_{2}} \cdot\left(l_{1}+l_{2}\right)\right)
\end{array}\right|=0 .
$$

The determinant (H.1) yields the following characteristic secular equation of sixth order for the above determined numerical values in Section 5:

$$
0.560 \cdot \lambda^{6}+7.073 \cdot \lambda^{4}-15.879 \cdot \lambda^{2}-23.602=0 .
$$

The numerical solution of the secular equation (H.2) gives the following set of roots:

$$
\lambda=\{ \pm 1.676 ; \pm 1.021 \cdot i ; \pm 3.794 \cdot i\} .
$$

\section{Generalized Forces of the Guided Mechanical System}

Calculation of the generalized forces $Q_{\delta\left(x_{1}\right)}, Q_{\delta\left(x_{2}\right)}$, and $Q_{\delta(\alpha)}$ in (J.1)-(J.3) requires the estimation of isochronous variation $\delta\left(z_{C_{3}}\right)$ of the vertical coordinate $z_{C_{3}}$ on the basis of approximate formula (D.2). The second equation of formulae (D.2) yields the following approximate expression for total ordinary differential of the vertical coordinate $z_{\mathrm{C}_{3}}$ :

$$
d\left(z_{\mathrm{C}_{3}}\right) \approx\left(l_{1} \cdot \alpha\right) \cdot d(\alpha)+\left(\left(\frac{a}{2}\right) \cdot \cos \left(\gamma_{0}\right)\right) \cdot d(\delta) .
$$

It is possible to get an approximate expression for total ordinary differential $d(\delta)$ in (I.1) on the basis of the previously derived formula (5), which produces the following equation:

$$
\begin{aligned}
& d(\delta) \approx \frac{1}{\left(a \cdot l_{2} \cdot \cos \left(\gamma_{0}\right)\right)}\left(\left(x_{1}-x_{2}+l_{1} \alpha\right)\right. \\
& \left.\quad \cdot\left(d\left(x_{1}\right)-d\left(x_{2}\right)+l_{1} \cdot d(\alpha)\right)-l_{1} \cdot l_{2} \cdot \alpha \cdot d(\alpha)\right) .
\end{aligned}
$$

Substitution of (I.2) into (I.1) results in the following expression:

$$
\begin{aligned}
& d\left(z_{C_{3}}\right) \approx\left(l_{1} \cdot \alpha\right) \cdot d(\alpha)+\frac{\left((a / 2) \cdot \cos \left(\gamma_{0}\right)\right)}{\left(a \cdot l_{2} \cdot \cos \left(\gamma_{0}\right)\right)} \\
& \quad \cdot\left(\left(x_{1}-x_{2}+l_{1} \alpha\right) \cdot\left(d\left(x_{1}\right)-d\left(x_{2}\right)+l_{1} \cdot d(\alpha)\right)\right. \\
& \left.\quad-l_{1} \cdot l_{2} \cdot \alpha \cdot d(\alpha)\right) .
\end{aligned}
$$

The formal change of differential symbol $d$ to the symbol of virtual displacement $\delta$ in (I.3) corresponds to imaginary freezing of time variable $t$ and gives the following expression for the isochronous variation $\delta\left(z_{\mathrm{C}_{3}}\right)$ of the vertical coordinate $z_{C_{3}}$ :

$$
\begin{array}{r}
\delta\left(z_{C_{3}}\right) \approx\left(l_{1} \cdot \alpha\right) \cdot \delta(\alpha)+\frac{1}{\left(2 \cdot l_{2}\right)} \cdot\left(\left(x_{1}-x_{2}+l_{1} \alpha\right)\right. \\
\left.\cdot\left(\delta\left(x_{1}\right)-\delta\left(x_{2}\right)+l_{1} \cdot \delta(\alpha)\right)-l_{1} \cdot l_{2} \cdot \alpha \cdot \delta(\alpha)\right),
\end{array}
$$


where $\delta\left(x_{1}\right) ; \delta\left(x_{2}\right)$; and $\delta(\alpha)$ are the three independent isochronous variations of the three generalized coordinates $x_{1} ; x_{2} ;$ and $\alpha$, which determine the current position of a $3 \mathrm{DOF}$ mechanical system in Figures 2 and 6.

Calculation of the first generalized force $Q_{\delta\left(x_{1}\right)}$ requires the imposition of the first independent virtual displacement $\delta\left(x_{1}\right) \neq 0$ to the mechanical system in Figure 6 with simultaneous "freezing" or "sticking down" of cart $B$ to the upper rails and "freezing" or "sticking to the left" of cable AE to the vertical axis $z$ factually assuming zero-values of another two virtual displacements $\delta\left(x_{2}\right)=0$ and $\delta(\alpha)=0$ :

$$
\begin{aligned}
Q_{\delta\left(x_{1}\right)} & =\left.\left(\frac{1}{\delta\left(x_{1}\right)}\right) \cdot\left(\left(\sum_{k} \delta A_{k}^{a}\right)_{\delta\left(x_{1}\right)}\right)\right|_{\delta\left(x_{2}\right)=0 ; \delta(\alpha)=0} \\
& =\frac{F_{1} \cdot \delta\left(x_{1}\right)+\left(\delta A_{1}\left(\mathbf{m}_{3} \mathbf{g}\right)\right)_{\delta\left(x_{1}\right)}}{\delta\left(x_{1}\right)} \\
Q_{\delta\left(x_{1}\right)} & =\frac{F_{1} \cdot \delta\left(x_{1}\right)+(-1) \cdot\left(m_{3} g\right) \cdot\left(\delta\left(z_{C_{3}}\right)\right)_{\delta\left(x_{1}\right)}}{\delta\left(x_{1}\right)}
\end{aligned}
$$

where the virtual work of the force $F_{2}$ in (I.5)-(I.6) at the virtual displacement $\delta\left(x_{1}\right) \neq 0$ has a zero-value because in our imagination we made "freezing" or "sticking down" of the cart $B$ to the upper rails with $\delta\left(x_{2}\right)=0$.

The second term $\left(\delta A_{1}\left(\mathbf{m}_{3} \mathbf{g}\right)\right)_{\delta\left(x_{1}\right)}$ in the numerator of (I.5) is the virtual work of the gravity force $\mathbf{m}_{3} \mathbf{g}$ of the crossbeam ED in the case of $\delta\left(x_{1}\right) \neq 0$ with $\delta\left(x_{2}\right)=0$ and $\delta(\alpha)=0$. The minus sign in the numerator of (I.6) shows that gravity force $\mathbf{m}_{\mathbf{3}} \mathbf{g}$ has negative work due to lifting up the point $C_{3}$ in this case in Figure 6. It is possible to calculate the algebraic expression of the isochronous variation $\left(\delta\left(z_{\mathrm{C}_{3}}\right)\right)_{\delta\left(x_{1}\right)}$ in the present case through the substitution of $\delta\left(x_{2}\right)=0$ and $\delta(\alpha)=0$ into (I.4):

$$
\begin{aligned}
\left(\delta\left(z_{C_{3}}\right)\right)_{\delta\left(x_{1}\right)}= & \left.\left(\delta\left(z_{C_{3}}\right)\right)\right|_{\delta\left(x_{2}\right)=0 ; \delta(\alpha)=0} \\
\approx & \frac{1}{\left(2 \cdot l_{2}\right)} \\
& \cdot\left(\left(x_{1}-x_{2}+l_{1} \alpha\right) \cdot\left(\delta\left(x_{1}\right)\right)\right) .
\end{aligned}
$$

Substitution of (I.7) into (I.6) results in the following expression:

$$
\begin{aligned}
& Q_{\delta\left(x_{1}\right)} \\
& \approx \frac{F_{1} \cdot \delta\left(x_{1}\right)+(-1) \cdot\left(m_{3} g\right) \cdot 1 /\left(2 \cdot l_{2}\right) \cdot\left(x_{1}-x_{2}+l_{1} \alpha\right) \cdot \delta\left(x_{1}\right)}{\delta\left(x_{1}\right)} .
\end{aligned}
$$

After simplifications (I.8) produces that

$$
Q_{\delta\left(x_{1}\right)} \approx F_{1}-\left(m_{3} g\right) \cdot \frac{1}{\left(2 \cdot l_{2}\right)} \cdot\left(x_{1}-x_{2}+l_{1} \alpha\right) .
$$

Derived expression (I.9) generalizes previous formula (E.2) in the case of $F_{1} \neq 0$. Formula (I.9) has been independently derived through virtual displacements. Similarity of expressions (E.2) and (I.9) proves the correctness of the proposed mathematical model. Substitution of $F_{1}=0$ into (I.9) again yields (E.2) that proves and confirms the correctness of (E.2).

Calculation of the second generalized force $Q_{\delta\left(x_{2}\right)}$ requires the imposition of the second independent virtual displacement $\delta\left(x_{2}\right) \neq 0$ to the mechanical system in Figure 6 with simultaneous "freezing" or "sticking down" of cart $A$ to the lower rails and "freezing" or "sticking to the left" of cable $\mathrm{AE}$ to the vertical axis $z$ factually assuming zero-values of another two virtual displacements $\delta\left(x_{1}\right)=0$ and $\delta(\alpha)=0$ :

$$
\begin{aligned}
Q_{\delta\left(x_{2}\right)}= & \left(\frac{1}{\delta\left(x_{2}\right)}\right) \\
& \left.\cdot\left(\left(\sum_{k} \delta A_{k}^{a}\right)_{\delta\left(x_{2}\right)}\right)\right|_{\delta\left(x_{1}\right)=0 ; \delta(\alpha)=0} \\
= & \frac{F_{2} \cdot \delta\left(x_{2}\right)+\left(\delta A_{2}\left(\mathbf{m}_{3} \mathbf{g}\right)\right)_{\delta\left(x_{2}\right)}}{\delta\left(x_{2}\right)} \\
Q_{\delta\left(x_{2}\right)}= & \frac{F_{2} \cdot \delta\left(x_{2}\right)+(-1) \cdot\left(m_{3} g\right) \cdot\left(\delta\left(z_{C_{3}}\right)\right)_{\delta\left(x_{2}\right)}}{\delta\left(x_{2}\right)},
\end{aligned}
$$

where the virtual work of the force $F_{1}$ in (I.10)-(I.11) at the virtual displacement $\delta\left(x_{2}\right) \neq 0$ has a zero-value because in our imagination we made "freezing" or "sticking down" of the cart $A$ to the lower rails with $\delta\left(x_{1}\right)=0$.

The second term $\left(\delta A_{2}\left(\mathbf{m}_{3} \mathbf{g}\right)\right)_{\delta\left(x_{2}\right)}$ in the numerator of (I.10) is the virtual work of the gravity force $\mathbf{m}_{\mathbf{3}} \mathbf{g}$ of the crossbeam ED in the case of $\delta\left(x_{2}\right) \neq 0$ with $\delta\left(x_{1}\right)=0$ and $\delta(\alpha)=0$. The minus sign in the numerator of (I.11) shows that gravity force $\mathbf{m}_{\mathbf{3}} \mathbf{g}$ has negative work due to lifting up the point $C_{3}$ in this case in Figure 6. It is possible to calculate the algebraic expression of the isochronous variation $\left(\delta\left(z_{C_{3}}\right)\right)_{\delta\left(x_{2}\right)}$ in the present case through the substitution of $\delta\left(x_{1}\right)=0$ and $\delta(\alpha)=0$ into (I.4):

$$
\begin{aligned}
\left(\delta\left(z_{C_{3}}\right)\right)_{\delta\left(x_{2}\right)}= & \left.\left(\delta\left(z_{C_{3}}\right)\right)\right|_{\delta\left(x_{1}\right)=0 ; \delta(\alpha)=0} \\
\approx & \frac{1}{\left(2 \cdot l_{2}\right)} \\
& \cdot\left(\left(x_{1}-x_{2}+l_{1} \alpha\right) \cdot\left(-\delta\left(x_{2}\right)\right)\right) .
\end{aligned}
$$


Substitution of (I.12) into (I.11) results in the following expression:

$$
Q_{\delta\left(x_{2}\right)} \approx \frac{F_{2} \cdot \delta\left(x_{2}\right)+(-1) \cdot\left(m_{3} g\right) \cdot 1 /\left(2 \cdot l_{2}\right) \cdot\left(x_{1}-x_{2}+l_{1} \alpha\right) \cdot(-1) \cdot \delta\left(x_{2}\right)}{\delta\left(x_{2}\right)} .
$$

After simplifications (I.13) produces that

$$
Q_{\delta\left(x_{2}\right)} \approx F_{2}+\left(m_{3} g\right) \cdot \frac{1}{\left(2 \cdot l_{2}\right)} \cdot\left(x_{1}-x_{2}+l_{1} \alpha\right) \text {. }
$$

Derived expression (I.14) generalizes previous formula (E.4) in the case of $F_{2} \neq 0$. Formula (I.14) has been independently derived through virtual displacements. Similarity of expressions (E.4) and (I.14) proves the correctness of proposed mathematical model. Substitution of $F_{2}=0$ into (I.14) yields again (E.4) that proves and confirms the correctness of (E.4).

Calculation of the third generalized force $Q_{\delta(\alpha)}$ requires the imposition of the third independent virtual displacement $\delta(\alpha) \neq 0$ to the mechanical system in Figure 6 with simultaneous "freezing" or "sticking down" of cart $A$ to the lower rails and "freezing" or "sticking down" of cart $B$ to the upper rails factually assuming zero-values of another two virtual displacements $\delta\left(x_{1}\right)=0$ and $\delta\left(x_{2}\right)=0$ :

$$
\begin{aligned}
Q_{\delta(\alpha)} & =\left.\left(\frac{1}{\delta(\alpha)}\right) \cdot\left(\left(\sum_{k} \delta A_{k}^{a}\right)_{\delta(\alpha)}\right)\right|_{\delta\left(x_{1}\right)=0 ; \delta\left(x_{2}\right)=0} \\
& =\frac{\left(\delta A_{3}\left(\mathbf{m}_{\mathbf{3}} \mathbf{g}\right)\right)_{\delta(\alpha)}}{\delta(\alpha)}
\end{aligned}
$$

$$
Q_{\delta(\alpha)} \approx \frac{(-1) \cdot\left(m_{3} g\right) \cdot\left(\left(l_{1} \cdot \alpha\right) \cdot \delta(\alpha)+1 /\left(2 \cdot l_{2}\right) \cdot\left(\left(x_{1}-x_{2}+l_{1} \alpha\right) \cdot\left(l_{1} \cdot \delta(\alpha)\right)-l_{1} \cdot l_{2} \cdot \alpha \cdot \delta(\alpha)\right)\right)}{\delta(\alpha)} .
$$

After simplifications (I.18) produces that

$$
\begin{gathered}
Q_{\delta(\alpha)} \approx(-1) \cdot\left(m_{3} g\right) \cdot\left(\left(l_{1} \cdot \alpha\right)+\frac{1}{\left(2 \cdot l_{2}\right)}\right. \\
\left.\cdot\left(l_{1} \cdot\left(x_{1}-x_{2}+l_{1} \alpha\right)-l_{1} \cdot l_{2} \cdot \alpha\right)\right) .
\end{gathered}
$$

The derived expression (I.19) is completely identical to previous formula (E.6). Formula (I.19) has been independently derived through virtual displacements. Coincidence of expressions (E.6) and (I.19) proves the correctness of the proposed mathematical model.

$$
Q_{\delta(\alpha)}=\frac{(-1) \cdot\left(m_{3} g\right) \cdot\left(\delta\left(z_{C_{3}}\right)\right)_{\delta(\alpha)}}{\delta(\alpha)},
$$

where the virtual works of the forces $F_{1}$ and $F_{2}$ in (I.15)-(I.16) at the virtual displacement $\delta(\alpha) \neq 0$ have zero-values because in our imagination we made "freezing" or "sticking down" of cart $A$ to the lower rails and "freezing" or "sticking down" of cart $B$ to the upper rails with $\delta\left(x_{1}\right)=0$ and $\delta\left(x_{2}\right)=0$.

The term $\left(\delta A_{3}\left(\mathbf{m}_{\mathbf{3}} \mathbf{g}\right)\right)_{\delta(\alpha)}$ in the numerator of (I.15) is the virtual work of the gravity force $\mathbf{m}_{\mathbf{3}} \mathbf{g}$ of the cross-beam ED in the case of $\delta(\alpha) \neq 0$ with $\delta\left(x_{1}\right)=0$ and $\delta\left(x_{2}\right)=0$. The minus sign in the numerator of (I.16) shows that gravity force $\mathbf{m}_{\mathbf{3}} \mathbf{g}$ has negative work due to lifting up the point $C_{3}$ in this case in Figure 6. It is possible to calculate the algebraic expression of the isochronous variation $\left(\delta\left(z_{C_{3}}\right)\right)_{\delta(\alpha)}$ in the present case through the substitution of $\delta\left(x_{1}\right)=0$ and $\delta\left(x_{2}\right)=0$ into (I.4):

$$
\begin{aligned}
& \left(\delta\left(z_{C_{3}}\right)\right)_{\delta(\alpha)}=\left.\left(\delta\left(z_{C_{3}}\right)\right)\right|_{\delta\left(x_{1}\right)=0 ; \delta\left(x_{2}\right)=0} \\
& \approx\left(l_{1} \cdot \alpha\right) \cdot \delta(\alpha)+\frac{1}{\left(2 \cdot l_{2}\right)} \\
& \quad \cdot\left(\left(x_{1}-x_{2}+l_{1} \alpha\right) \cdot\left(l_{1} \cdot \delta(\alpha)\right)-l_{1} \cdot l_{2} \cdot \alpha \cdot \delta(\alpha)\right) .
\end{aligned}
$$

Substitution of (I.17) into (I.16) results in the following expression:

\section{J. Lagrange Equations of the Guided Mechanical System}

Application of independent control forces $F_{1}=u_{1}(t)[\mathrm{N}]$ and $F_{2}=u_{2}(t)[\mathrm{N}]$ requires the estimation of new values of three generalized forces $Q_{\delta x_{1}}, Q_{\delta x_{2}}$, and $Q_{\delta \alpha}$ in the right-hand sides of the following Lagrange equations for Figure 6:

$$
\begin{gathered}
\frac{d}{d t}\left(\frac{\partial T}{\partial \dot{x}_{1}}\right)-\frac{\partial T}{\partial x_{1}}=Q_{\delta\left(x_{1}\right)} ; \\
\frac{d}{d t}\left(\frac{\partial T}{\partial \dot{x}_{2}}\right)-\frac{\partial T}{\partial x_{2}}=Q_{\delta\left(x_{2}\right)} ; \\
\frac{d}{d t}\left(\frac{\partial T}{\partial \dot{\alpha}}\right)-\frac{\partial T}{\partial \alpha}=Q_{\delta(\alpha)},
\end{gathered}
$$


where magnitudes of generalized forces $Q_{\delta\left(x_{1}\right)}, Q_{\delta\left(x_{2}\right)}$, and $Q_{\delta(\alpha)}$ are determined by the action of three independent external forces $F_{1}=u_{1}(t)[\mathrm{N}], F_{2}=u_{2}(t)[\mathrm{N}]$, and $m_{3} g[\mathrm{~N}]$ (Figure 6). The left-hand sides of Lagrange equations (J.1)(J.3) are determined by (G.5), (G.11), and (G.17).

Substitution of (G.5) and (I.9) into (J.1) results in the following first Lagrange equation for the guided mechanical system:

$$
\begin{aligned}
& \left(m_{1}+m_{3}\right) \cdot\left(\frac{d^{2} x_{1}}{d t^{2}}\right)+m_{3} \cdot l_{1} \cdot\left(\frac{d^{2} \alpha}{d t^{2}}\right) \\
& \approx F_{1}-\left(m_{3} g\right) \cdot \frac{1}{\left(2 \cdot l_{2}\right)} \cdot\left(x_{1}-x_{2}+l_{1} \alpha\right) .
\end{aligned}
$$

Substitution of (G.11) and (I.14) into (J.2) results into the following second Lagrange equation for the guided mechanical system:

$$
\begin{aligned}
& m_{2} \cdot\left(\frac{d^{2} x_{2}}{d t^{2}}\right) \approx F_{2}+\left(m_{3} g\right) \cdot \frac{1}{\left(2 \cdot l_{2}\right)} \\
& \cdot\left(x_{1}-x_{2}+l_{1} \alpha\right) \text {. }
\end{aligned}
$$

Substitution of (G.17) and (I.19) into (J.3) results into the following third Lagrange equation for the guided mechanical system:

$$
\begin{aligned}
& m_{3} \cdot l_{1}^{2} \cdot\left(\frac{d^{2} \alpha}{d t^{2}}\right)+m_{3} \cdot l_{1} \cdot\left(\frac{d^{2} x_{1}}{d t^{2}}\right) \approx(-1) \cdot\left(m_{3} g\right) \\
& \cdot\left(\left(l_{1} \cdot \alpha\right)+\frac{1}{\left(2 \cdot l_{2}\right)}\right. \\
& \left.\cdot\left(l_{1} \cdot\left(x_{1}-x_{2}+l_{1} \alpha\right)-l_{1} \cdot l_{2} \cdot \alpha\right)\right) .
\end{aligned}
$$

\section{Nomenclature}

\section{DOF: Degree of freedom}

ODE: Ordinary differential equation

point $A$ : Point of bridge crane 1 position

point $B$ : Point of bridge crane 2 position

DE: $\quad$ Cross-beam 3

point $C_{3}$ : Point of gravity center of cross-beam 3

$g$ : Scalar value of gravitational acceleration $\left(\mathrm{m} / \mathrm{s}^{2}\right)$

$t: \quad$ Current time (s)

$l_{1}$ : $\quad$ Length of the left cable AE, that is,

$l_{\mathrm{AE}}=l_{1}(\mathrm{~m})$

$l_{2}$ : Length of the right cable $\mathrm{BD}$, that is, $l_{\mathrm{BD}}=l_{2}(\mathrm{~m})$

$a$ Length of cross-beam DE (m)

$b$ : $\quad$ Vertical distance between the carts $A$ and $B(\mathrm{~m})$

$x_{1}$ : The horizontal coordinate of bridge crane p. $A(\mathrm{~m})$

$d x_{1} / d t$ : The horizontal projection of bridge crane p. $A$ velocity $(\mathrm{m} / \mathrm{s})$ $d^{2} x_{1} / d t^{2}: \quad$ The horizontal projection of bridge crane $\mathrm{p}$. $A$ acceleration $\left(\mathrm{m} / \mathrm{s}^{2}\right)$

$x_{2}$ : The horizontal coordinate of bridge crane $\mathrm{p}$. $B(\mathrm{~m})$

$d x_{2} / d t: \quad$ The horizontal projection of bridge crane $\mathrm{p}$. $B$ velocity $(\mathrm{m} / \mathrm{s})$

$d^{2} x_{2} / d t^{2}: \quad$ The horizontal projection of bridge crane $\mathrm{p}$. $B$ acceleration $\left(\mathrm{m} / \mathrm{s}^{2}\right)$

$x_{C_{3}}$ : The horizontal coordinate of p. $C_{3}(\mathrm{~m})$

$d\left(x_{C_{3}}\right) / d t$ : The horizontal projection of $\mathrm{p} . \mathrm{C}_{3}$ velocity $(\mathrm{m} / \mathrm{s})$

$d^{2}\left(x_{C_{3}}\right) / d t^{2}$ : The horizontal projection of p. $C_{3}$ acceleration $\left(\mathrm{m} / \mathrm{s}^{2}\right)$

$z_{C_{3}}: \quad$ The vertical coordinate of p. $C_{3}(\mathrm{~m})$

$d\left(z_{C_{3}}\right) / d t$ : The vertical projection of p. $C_{3}$ velocity $(\mathrm{m} / \mathrm{s})$

$d^{2}\left(z_{C_{3}}\right) / d t^{2}$ : The vertical projection of $\mathrm{p} . C_{3}$ acceleration $\left(\mathrm{m} / \mathrm{s}^{2}\right)$

$\alpha: \quad$ The angular distance between cable AE and vertical line ( $\mathrm{rad})$

$d \alpha / d t$ : The angular velocity of cable AE around p. A ( $\mathrm{rad} / \mathrm{s})$

$d^{2} \alpha / d t^{2}$ : The angular acceleration of cable AE around p. $A\left(\mathrm{rad} / \mathrm{s}^{2}\right)$

$\beta$ : $\quad$ The angular distance between cable BD and vertical line ( $\mathrm{rad}$ )

$d \beta / d t$ : The angular velocity of cable $\mathrm{BD}$ around p. $B$ $(\mathrm{rad} / \mathrm{s})$

$d^{2} \beta / d t^{2}$ : The angular acceleration of cable BD around p. $B\left(\mathrm{rad} / \mathrm{s}^{2}\right)$

$\gamma: \quad$ The inclination angle of cross-beam DE with horizontal line (rad)

$d \gamma / d t: \quad$ The angular velocity of cross-beam DE around horizontal line or around p. $E(\mathrm{rad} / \mathrm{s})$

$d^{2} \gamma / d t^{2}: \quad$ The angular acceleration of cross-beam DE around horizontal line or around p. E $\left(\mathrm{rad} / \mathrm{s}^{2}\right)$

$m_{1}$ : $\quad$ Mass of bridge crane p. $A(\mathrm{~kg})$

$m_{2}$ : $\quad$ Mass of bridge crane p. $B(\mathrm{~kg})$

$m_{3}$ : $\quad$ Mass of cross-beam DE $(\mathrm{kg})$

$T$ : $\quad$ Kinetic energy of the system "heavy crossbeam, two moving carts" $(\mathrm{J}=\mathrm{N}-\mathrm{m})$

$\Pi$ : $\quad$ Potential energy of the system "heavy crossbeam, two moving carts" $(\mathrm{J}=\mathrm{N}-\mathrm{m})$

$Q_{x_{1}} ; Q_{x_{2}} ; Q_{\alpha}:$ Generalized forces $(\mathrm{N})$

$\lambda_{i}$ : $\quad$ Roots of the secular equation

$k_{1} ; k_{2}$ : $\quad$ First and second natural frequencies of system oscillations $(1 / \mathrm{s})$

$\tau_{1} ; \tau_{2}: \quad$ First and second natural periods of system oscillations (s).

\section{Additional Points}

Highlights. A new 2D model of a 3DOF system "two moving bridge cranes, transported cross-beam" was proposed. A new open loop optimal control problem was formulated and solved with JModelica.org freeware. Simple engineering methodology for optimal control realization was proposed. 


\section{Disclosure}

The submission of the authors' paper implies that it has not been previously published, that it is not under consideration for publication elsewhere, and that it will not be published elsewhere in the same form without the written permission of the editors.

\section{Competing Interests}

The authors Alexander V. Perig, Alexander N. Stadnik, Alexander A. Kostikov, and Sergey V. Podlesny declare that there is no conflict of interests regarding the publication of this paper.

\section{Authors' Contributions}

All authors participated in the design of this work and performed equally. All authors read and approved the final manuscript.

\section{References}

[1] E. M. Abdel-Rahman, A. H. Nayfeh, and Z. N. Masoud, "Dynamics and control of cranes: a review," Journal of Vibration and Control, vol. 9, no. 7, pp. 863-908, 2003.

[2] M. S. A. Deen Ali, N. R. Babu, and K. Varghese, "Collision free path planning of cooperative crane manipulators using genetic algorithm," Journal of Computing in Civil Engineering, vol. 19, no. 2, pp. 182-193, 2005.

[3] A. Arena, A. Casalotti, W. Lacarbonara, and M. P. Cartmell, "Dynamics of container cranes: three-dimensional modeling, full-scale experiments, and identification," International Journal of Mechanical Sciences, vol. 93, pp. 8-21, 2015.

[4] M. P. Cartmell, L. Morrish, and A. J. Taylor, "Dynamics of spreader motion in a gantry crane," Proceedings of the Institution of Mechanical Engineers, Part C: Journal of Mechanical Engineering Science, vol. 212, no. 2, pp. 85-105, 1998.

[5] G. Castelli, E. Ottaviano, and P. Rea, "A Cartesian CableSuspended Robot for improving end-users' mobility in an urban environment," Robotics and Computer-Integrated Manufacturing, vol. 30, no. 3, pp. 335-343, 2014.

[6] J.-H. Cha, S.-H. Ham, K.-Y. Lee, and M.-I. Roh, "Application of a topological modelling approach of multi-body system dynamics to simulation of multi-floating cranes in shipyards," Proceedings of the Institution of Mechanical Engineers, Part K: Journal of Multi-Body Dynamics, vol. 224, no. 4, pp. 365-373, 2010.

[7] K. Goodwin, "RoboCrane construction of bridges," Transportation Research Record, no. 1575, pp. 42-46, 1997.

[8] J. Huang, Z. Liang, and Q. Zang, "Dynamics and swing control of double-pendulum bridge cranes with distributedmass beams," Mechanical Systems and Signal Processing, vol. 5455, pp. 357-366, 2015.

[9] S. Lahouar, E. Ottaviano, S. Zeghoul, L. Romdhane, and M. Ceccarelli, "Collision free path-planning for cable-driven parallel robots," Robotics and Autonomous Systems, vol. 57, no. 11, pp. 1083-1093, 2009.

[10] L. Pigani and P. Gallina, "Cable-direct-driven-robot (CDDR) with a 3-link passive serial support," Robotics and ComputerIntegrated Manufacturing, vol. 30, no. 3, pp. 265-276, 2014.
[11] O. Sawodny, H. Aschemann, and S. Lahres, "An automated gantry crane as a large workspace robot," Control Engineering Practice, vol. 10, no. 12, pp. 1323-1338, 2002.

[12] J. Smoczek, "Fuzzy crane control with sensorless payload deflection feedback for vibration reduction," Mechanical Systems and Signal Processing, vol. 46, no. 1, pp. 70-81, 2014.

[13] B. Zi, B. Y. Duan, J. L. Du, and H. Bao, "Dynamic modeling and active control of a cable-suspended parallel robot," Mechatronics, vol. 18, no. 1, pp. 1-12, 2008.

[14] A. A. Kostikov, A. V. Perig, D. Y. Mikhieienko, and R. R. Lozun, "Numerical JModelica.org-based approach to a simulation of Coriolis effects on guided boom-driven payload swaying during non-uniform rotary crane boom slewing," Journal of the Brazilian Society of Mechanical Sciences and Engineering, vol. 39, no. 3, pp. 737-756, 2017.

[15] A. A. Kostikov, A. V. Perig, and R. R. Lozun, "Simulationassisted teaching of graduate students in transport: a case study of the application of acausal freeware JModelica.org to solution of Sakawa's open-loop optimal control problem for payload motion during crane boom rotation," International Journal of Mechanical Engineering Education, vol. 45, no. 1, pp. 3-27, 2017.

[16] A. V. Perig, A. N. Stadnik, and A. I. Deriglazov, "Spherical pendulum small oscillations for slewing crane motion," The Scientific World Journal, vol. 2014, Article ID 451804, 10 pages, 2014.

[17] A. V. Perig, A. N. Stadnik, A. I. Deriglazov, and S. V. Podlesny, "3 DOF spherical pendulum oscillations with a uniform slewing pivot center and a small angle assumption," Shock and Vibration, vol. 2014, Article ID 203709, 32 pages, 2014.

[18] J. G. Papastavridis, Analytical Mechanics. A Comprehensive Treatise on the Dynamics of Constrained Systems; for Engineers, Physicists, Oxford University Press, New York, NY, USA, 2001.

[19] A. B. Modelon, “JModelica.org User Guide: Version 1.17," 2015 http://www.jmodelica.org/api-docs/usersguide/JModelicaUsersGuide-1.17.0.pdf. 


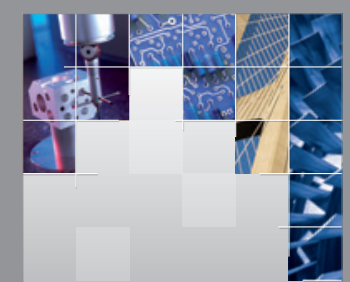

\section{Enfincering}
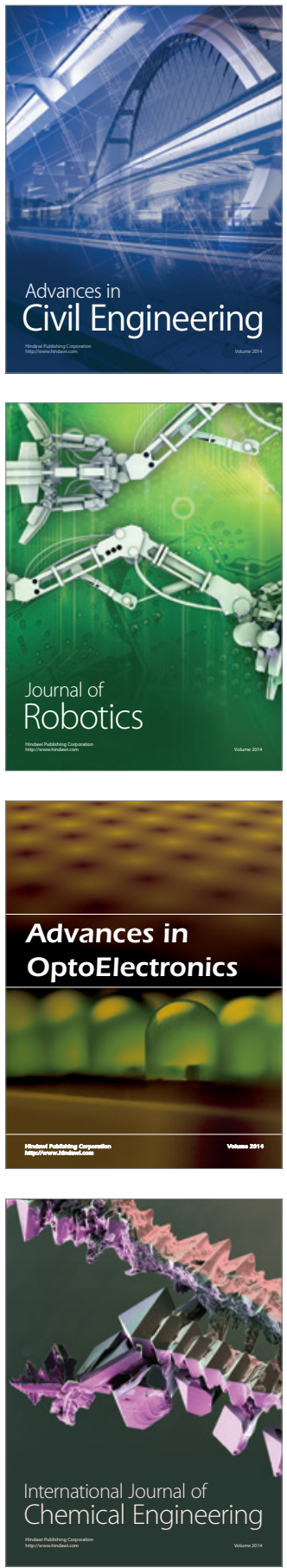

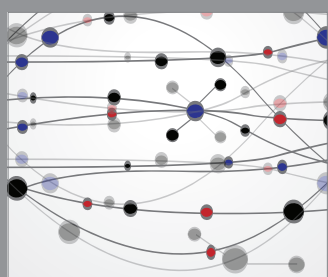

The Scientific World Journal

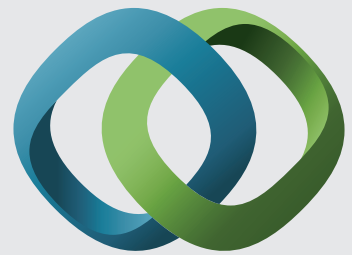

\section{Hindawi}

Submit your manuscripts at

https://www.hindawi.com
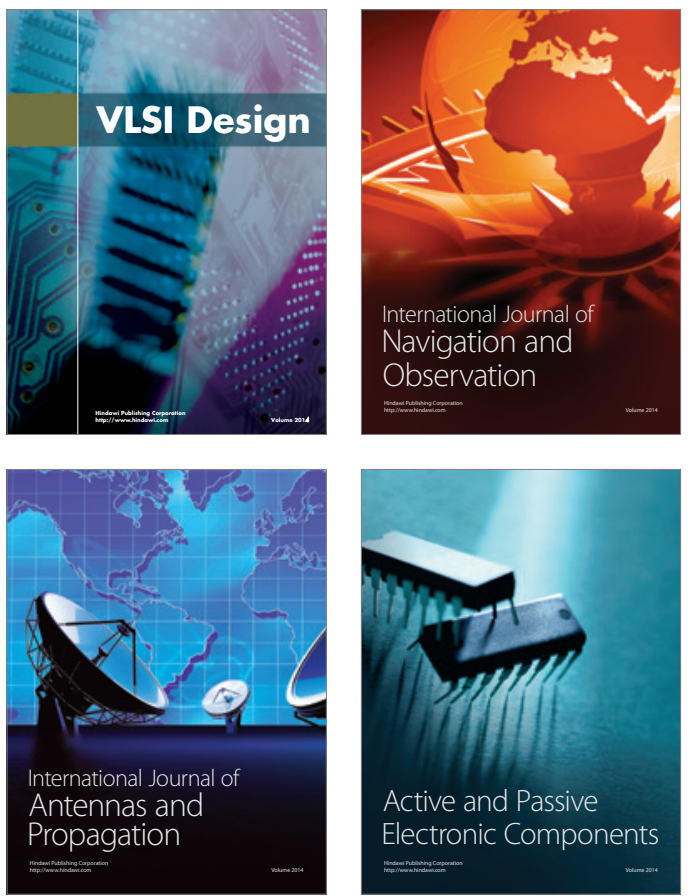
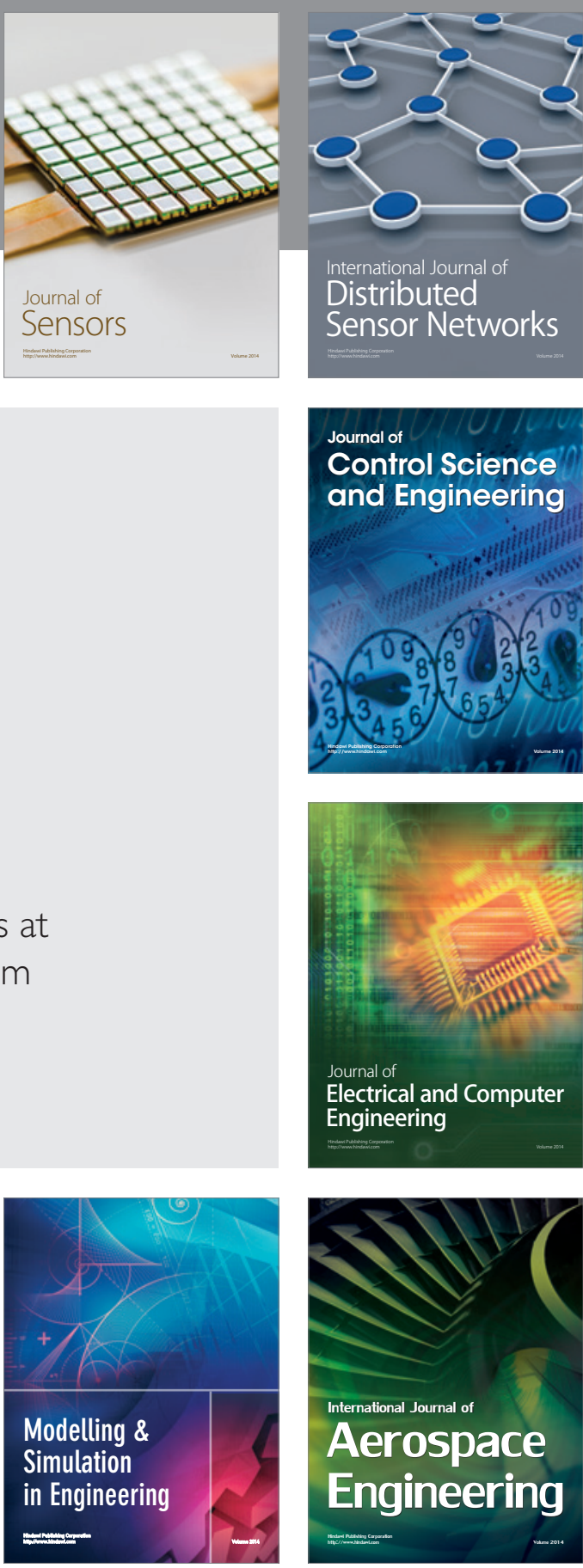

International Journal of

Distributed

Sensor Networks

$-$

Joumal of

Control Science

and Engineering
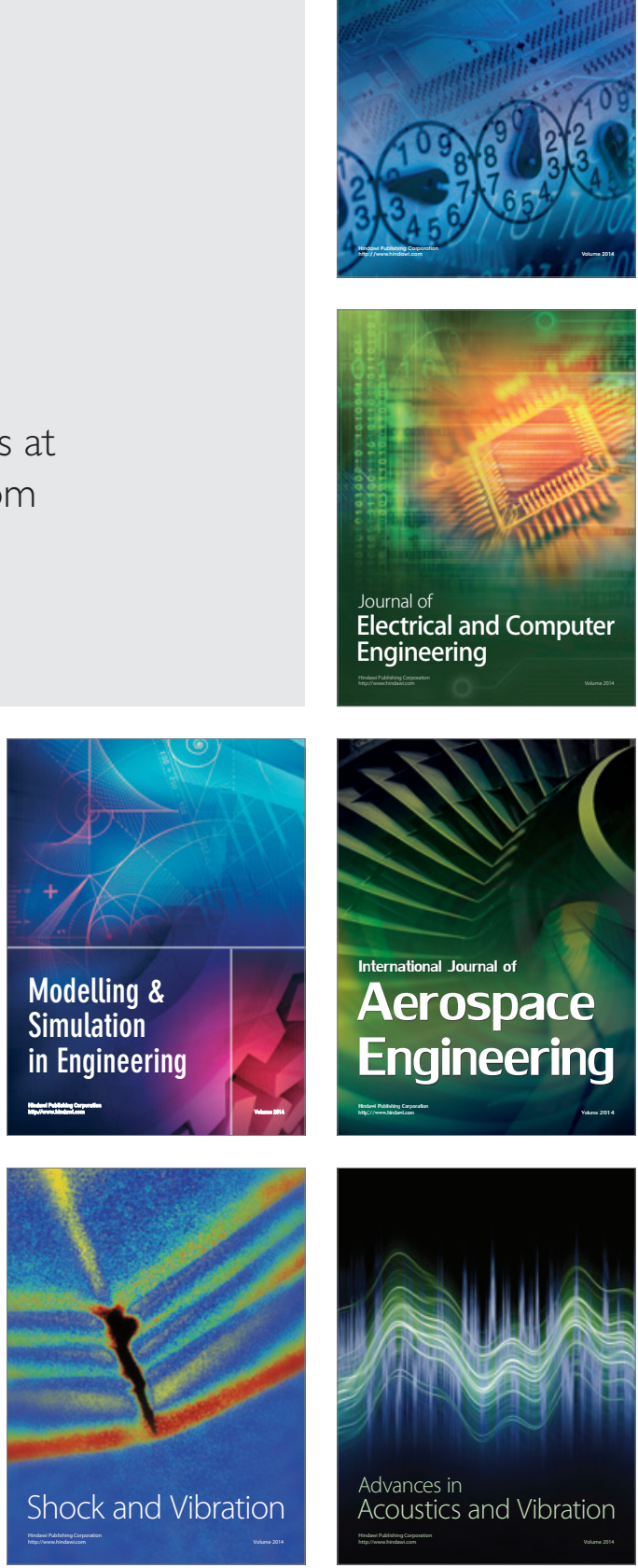